\title{
Interpretation of Explanatory Variables Impacts in Compositional Regression Models
}

\author{
Joanna Morais \\ Toulouse School \\ of Economics
}

\author{
Christine Thomas-Agnan \\ Toulouse School \\ of Economics
}

\author{
Michel Simioni \\ INRA, UMR 1110 \\ MOISA
}

\begin{abstract}
We are interested in modeling the impact of media investments on automobile manufacturer's market shares. Regression models have been developed for the case where the dependent variable is a vector of shares. Some of them, from the marketing literature, are easy to interpret but quite simple (Model A). Alternative models, from the compositional data analysis literature, allow a large complexity but their interpretation is not straightforward (Model B). This paper combines both approaches in order to obtain a performing market share model and develop relevant interpretations for practical use.

We prove that Model A is a particular case of Model B, and that an intermediate specification is possible (Model AB). A model selection procedure is proposed. Several impact measures are presented and we show that elasticities are particularly useful: they can be computed from the transformed or from the original model, and they are linked to the simplicial derivatives.
\end{abstract}

Keywords: elasticity, model selection, odds ratio, marginal effect, compositional model, compositional differential calculus, market-shares, media investments impact.

\section{Introduction}

We are interested in modeling the impact of media investments on the distribution of automobile manufacturer sales. We consider that the sales volume in a particular segment of the automobile market is mainly determined by the demand through the socio-economic and regulatory context. Thus, each brand tries to have "the largest share of the cake" using marketing tools, like price and media investments. The impact of media investments of brand $j$ on its own sales cannot be assessed without taking into account the competition. Thus, we want to model the impact of media investments on market-shares, taking into account the marketing actions of competitors, directly (cross-effects) or indirectly.

In the existing literature, we found different types of models to model shares (see Morais, Thomas-Agnan, and Simioni (2016) for a comparison). Some of them, from the marketing or econometric literature, are perfectly adapted to model market-shares and to interpret direct and cross impacts of media investments, but the proposed models are quite simple and do not allow the specification of cross effects between brands. Actually, a "fully extended attraction 
model" is proposed by Cooper and Nakanishi Cooper and Nakanishi (1988) to do so. However it does not succeed in identifying the parameters themselves but only their centered version (see Morais, Thomas-Agnan, and Simioni (2017) for details). Other models adapted to share data are proposed, which are called compositional regression models and are based on the simplicial geometry, a geometry initially developed in Aitchison (1986). These mathematical models are very flexible in terms of explanatory variables and complexity (alternative-specific and cross-effect parameters), but their interpretation is not straightforward. This paper combines both approaches in order to obtain a performing market-share model allowing to get relevant and appropriate interpretations, which can be used for example to help decision making of automobile manufacturers concerning their media investments.

Here we focus on compositional models which are coming from the so called Compositional Data Analysis (CODA) literature (see Pawlowsky-Glahn, Egozcue, and Tolosana-Delgado (2015)). A vector of $D$ shares is a composition of $D$ positive components for which only the relative information is of interest. It can be represented in a space called the simplex which is characterized by the scale invariance property. Compositional models are "transformation" models in the sense that they assume a distribution (generally Gaussian) for a log-ratio transformation of shares. Transformation models have several advantages compared to other share models: they are easy to estimate (usually by OLS on coordinates) and flexible in terms of explanatory variables (they can be compositional or non-compositional variables, with or without component-specific parameters). More specifically, we focus on models where a compositional dependent variable is explained by some compositional explanatory variables. We distinguish two specifications of this model: in Model A, a unique coefficient is associated to each compositional explanatory variable (see Wang, Shangguan, Wu, and Guan (2013)), whereas in Model B a compositional explanatory variable is associated to component-specific and cross-effect coefficients (see Chen, Zhang, and Li (2017)). We prove that Model A is a particular case of Model B, and that the two specifications can be mixed (Model AB) in order to attribute more or less complexity to some explanatory variable impacts. A model selection procedure is proposed using an adapted Fisher test, considering that Model B is the unconstrained model to be compared to the constrained models, Model A or Model AB.

In the CODA literature, the interpretation of parameters is not well developed. They are usually interpreted in terms of marginal effects on the transformed shares (see Muller, Hron, Fiserova, Smahaj, Cakirpaloglu, and Vancakova (2015) and Chen et al. (2017)). In this paper we propose several interpretations directly linked to the shares, in terms of marginal effects, elasticities and odds ratios, in order to enhance the interpretability of these models. We show that marginal effects on shares may not be well adapted to interpret these models because they depend a lot on the considered observation. Elasticities are useful to isolate the impact of an explanatory variable on a particular share as they correspond to the relative variation of a component with respect to the relative variation of an explanatory variable, ceteris paribus (in a simplex sense). We show that they can be computed from the transformed model or equivalently from the model in the simplex. Other types of elasticities and odds ratios can be computed for ratios of shares, which are observation independent but they can be complicated to use in practice.

Model A, Model B and an intermediate Model AB are applied to an automobile market data set, where the aim is to explain the brands market-shares in a segment with brands media investments. The models are interpreted using marginal effects, elasticities and odds ratios, and they are compared using the Fisher test and in terms of (out-of-sample) goodness-of-fit using quality measures adapted for share data.

This paper is organized as follows: the second section presents the two types of compositional models, their intermediate specification, along with the adapted Fisher test for model selection; the third section explains how to interpret them; the fourth section presents the results of the estimation of the models for the French automobile market along with interpretations, Fisher tests and quality measures. Finally, the last section concludes on the findings and on 
further directions to be investigated.

\section{Compositional regression models}

\subsection{Definition and notations}

By definition shares are "compositional data": a composition is a vector of $D$ parts of some whole which carries relative information. Compositions data are equivalence classes defined by the scale invariance propriety (Barcelo-Vidal and Martín-Fernández (2016)). A D-composition can be represented in a space called the simplex $\mathcal{S}^{D}$.

$$
\mathcal{S}^{D}=\left\{\mathbf{s}=\left(s_{1}, s_{2}, \ldots, s_{D}\right)^{\prime}: s_{j}>0, j=1, \ldots, D ; \sum_{j=1}^{D} s_{j}=1\right\}
$$

Therefore one needs to use distributions on the simplex in order to model compositional data, and classical regression models are not adapted to that case.

The following operations are used in the simplex (see van den Boogaart and Tolosana-Delgado (2013) for example):

- $\mathcal{C}()$ denotes the closure operation which represents the original volumes as shares (without loss of information from the CODA perspective):

$$
\mathcal{C}\left(\check{x}_{1}, \ldots, \check{x}_{D}\right)^{\prime}=\left(\frac{\check{x}_{1}}{\sum_{j=1}^{D} \check{x}_{j}}, \ldots, \frac{\check{x}_{D}}{\sum_{j=1}^{D} \check{x}_{j}}\right)^{\prime}=\left(x_{1}, \ldots, x_{D}\right)^{\prime}
$$

where $\check{x}$ denotes the volume and $x$ denotes the share of a variable.

- $\oplus$ is the perturbation operation, corresponding to the addition operation in the simplex:

$$
\mathbf{x} \oplus \mathbf{y}=\mathcal{C}\left(x_{1} y_{1}, \ldots, x_{D} y_{D}\right)^{\prime}
$$

with $\mathbf{x}, \mathbf{y} \in \mathcal{S}^{D}$

- $\odot$ is the power transformation, corresponding to the multiplication operation in the simplex:

$$
\lambda \odot \mathbf{x}=\mathcal{C}\left(x_{1}^{\lambda}, \ldots, x_{D}^{\lambda}\right)^{\prime}
$$

with $\lambda \in \mathbb{R}, \mathbf{x} \in \mathcal{S}^{D}$

- $\square$ is the compositional matrix product, corresponding to the matrix product in the simplex:

$$
\mathbf{B} \bullet \mathbf{x}=\mathcal{C}\left(\prod_{j=1}^{D_{X}} x_{j}^{b_{1 j}}, \ldots, \prod_{j=1}^{D_{X}} x_{j}^{b_{D_{S} j}}\right)^{\prime}
$$

with $\mathbf{B} \in \mathbb{R}_{D_{S}, D_{X}}, \mathbf{x} \in \mathcal{S}^{D}$

\subsection{Log-ratio transformation approach}

Compositional data analysis is based on using log-ratio transformations that result in real coordinates in the Euclidean space $R^{D-11}$. Then, classical methods suited for data in the Euclidean space, like linear regression models, can be used on coordinates. Below, terms with a "*" refer to transformed elements (in coordinates), whereas terms without "*" refer to elements in the simplex (compositions).

\footnotetext{
${ }^{1}$ Or in $\mathbb{R}^{D}$ in the case of the CLR transformation.
} 
Several transformations are developed in the CODA literature (see Egozcue, PawlowskyGlahn, Mateu-Figueras, and Barcelo-Vidal (2003) for example). The ILR (isometric logratio) transformation is preferred for compositional regression models, against the ALR (additive log-ratio) and the CLR (centered log-ratio) transformation (see van den Boogaart and Tolosana-Delgado (2013)). It consists in a projection of components on an orthonormal basis of $\mathcal{S}^{D}$ in order to obtain $D-1$ orthonormal coordinates. Considering the transformation matrix $\mathbf{V}_{D \times(D-1)}$ with columns $\mathbf{v}_{l}=\operatorname{clr}\left(\mathbf{e}_{l}\right)$ where $\mathbf{e}_{1}, \ldots, \mathbf{e}_{D-1}$ is an orthonormal basis of $\mathcal{S}^{D}$ such that $\mathbf{V}^{\prime} \mathbf{V}=\mathbf{I}_{D-1}$, ILR coordinates are defined as:

$$
\operatorname{ilr}(\mathbf{s})=\mathbf{V}^{\prime} \log (\mathbf{s})=\mathbf{s}^{*}=\left(s_{1}^{*}, \ldots, s_{D-1}^{*}\right)^{\prime}
$$

Its inverse transformation is given by: $\mathbf{S}=i l r^{-1}\left(\mathbf{S}^{*}\right)=\mathcal{C}\left(\exp \left(\mathbf{V} \mathbf{S}^{*}\right)\right)^{\prime}$.

After inverse transformation, results of a compositional analysis are the same regardless of the chosen transformation.

\subsection{Two types of compositional models}

In this section, we consider two types of models adapted to a compositional dependent variable explained by compositional explanatory variable (and potentially non-compositional variables). The difference between the two models is about the specification of the relationship between compositional explanatory and dependent variables: in contrast with Model B, Model A does not allow for component-specific and cross effect parameters associated to a compositional explanatory variable $\mathbf{X}$. In this paper, we add the possibility to use classical (noncompositional) variables $Z$ as explanatory variables. There is no difference between Models $\mathrm{A}$ and $\mathrm{B}$ with regard to classical variables: component-specific parameters are specified.

For simplicity, models are presented with a single explanatory variable of each type (compositional $\mathbf{X}$ and non-compositional $Z$ ), but of course several ones can be used like in the examples presented in Section 4.

Model A: Compositional dependent and explanatory variables without component-specific and cross-effect parameters

Model A is presented by Wang et al. (2013). In Model A, a compositional explanatory variable is associated to a unique parameter $b \in \mathbb{R}$ (see Table 1, equation (1)). Thus, cross-effects ${ }^{2}$ are not modeled directly, but indirectly through the shares closure. Indeed, we show in Morais et al. (2017) that Model A in equation (1) can be written in attraction form like in equation (3). This equation contains a closure, and we can see that a change of $X_{l}$ will have an indirect impact on $S_{j}$ through the denominator. Moreover, the attraction form of Model A enables to see that Model A respects the IIA (independence from irrelevant alternative) property. This property means that the ratio of shares of two alternatives $j$ and $l, S_{j} / S_{l}$, does not depend on characteristics of other alternatives $m \neq j, l$. Note that equation (3) can be expressed either in terms of shares $X_{j}$ or in terms of volumes $\check{X}_{j}$ thanks to the scale invariance property. If a non-compositional explanatory variable $Z$ is used in Model A, it is associated to a composition of parameters $\mathbf{c}^{3}$.

The ILR coordinates are used in order to estimate Model A (see equation (5)). Assuming that the transformed error terms are normal (implying that the non-transformed compositional error terms follow the normal distribution on the simplex; see Mateu-Figueras and PawlowskyGlahn (2008) for more details on this distribution), we can use OLS to estimate the model.

An important feature of Model $\mathbf{A}$ is that compositional explanatory variables $\mathbf{X}$ have to be of the same dimension that the compositional dependent variable $\mathbf{S}$, such that $\mathbf{S}, \mathbf{X} \in \mathcal{S}^{D}$.

\footnotetext{
${ }^{2}$ We denote by cross-effect the effect of a variation of $X_{l}$ on $S_{j}$, where $l \neq j$.

${ }^{3}$ It can be surprising to see that in the attraction form of Model A, the variable $Z$ is powering the intercept $c_{j}$, but this corresponds to the term $Z_{t} \odot \mathbf{c}$.
} 
This model is adapted when compositions $\mathbf{X}$ and $\mathbf{S}$ refer to two variables associated to the same components in the same order, for example $\mathbf{S}$ can be the composition of brands marketshares and $\mathbf{X}$ the composition of brand media investments (where brands are in the same order in $\mathbf{S}$ and $\mathbf{X}$ ) (see Section 4), or $\mathbf{S}$ can be the composition of GDP from three sectors and $\mathbf{X}$ the composition of labor force of these three sectors. Otherwise, this model makes no sense. Then, equation (5) is estimated using $(D-1) \times T$ observations (the number of ILR coordinates $D-1$ times the number of observations $T)$. Actually, this model specification is close to the specification of multinomial or market-share models (see Morais et al. (2016) for a comparison).

Model B: Compositional dependent and explanatory variables with component-specific and cross-effect parameters

Model B is used by van den Boogaart and Tolosana-Delgado (2013) and Chen et al. (2017) for example. Using exactly the same dependent and explanatory variables as Model A (see equation (2)), it allows each component $X_{l}$ of $\mathbf{X}$ to have a specific impact on each component $S_{j}$ of $\mathbf{S}$. This is particularly visible in the attraction form of Model B (equation (4)): instead of having a unique parameter $b \in \mathbb{R}$ associated to $\mathbf{X}$, we have a matrix of parameters $\mathbf{B} \in$ $\mathbb{R}_{D_{S}, D_{X}}$. If $D_{S}=D_{X}$ and $\mathbf{S}$ and $\mathbf{X}$ refer to the same components in the same order, then $\mathbf{B}$ is a square matrix with direct effect on the diagonal and cross-effects outside of the diagonal. There is no difference between Model A and Model B for the specification of the intercept and non-compositional explanatory variables. The same remark as for Model A can be done concerning the attraction form of Model B: equation (4) can be expressed either in terms of shares $X_{j}$ or in terms of volumes $\check{X}_{j}$ thanks to the scale invariance property.

As in Model A, in order to estimate Model B, we transform it using the ILR transformation (see equation (6)). But here, $D_{S}-1$ equations are estimated separately (one for each coordinate of $\mathbf{S}$ ) with $T$ observations each. The complexity of Model B is reflected by a large number of parameters. This can be an issue if the number of observations $T$ is too small.

Note that in Model $\mathrm{B}, \mathbf{X} \in \mathcal{S}^{D_{X}}$ and $\mathbf{S} \in \mathcal{S}^{D_{S}}$ can have different dimensions. For example, $\mathbf{S}$ can be the composition of GDP from three sectors and $\mathbf{X}$ the composition of labor force for six occupation categories. In our application, $D_{S}=D_{X}: \mathbf{S}$ is the composition of brands market-shares and $\mathbf{X}$ is the composition of brand media investments (see Section 4).

\section{Intermediate specification (Model AB) and model selection}

One can show that Model A is a particular case of Model B where $D_{S}=D_{X}$ and where $B^{*}$ is a diagonal matrix with $b^{*}=b$ on the diagonal and 0 otherwise, that is where only the $j^{\text {th }}$ ILR coordinates of compositional explanatory variables are relevant to explain the $j^{\text {th }}$ ILR coordinates of the dependent variable (see the appendix A.1 for a proof in the case of $D=3$ ). Then, in a given model it is possible to mix the two specifications if and only if $D_{S}=D_{X}$ for the explanatory variable with the Model A specification. This model called Model AB is defined as follows in the simplex:

$$
\mathbf{S}_{t}=\mathbf{a} \oplus \beta \odot \mathbf{X}_{t} \oplus \mathbf{B} \odot \mathbf{Y}_{t} \oplus Z_{t} \odot \mathbf{c} \oplus \epsilon
$$

Model AB can be estimated using its expression in ILR coordinates:

$$
S_{j t}^{*}=\sum_{d=1}^{D-1} \nVdash_{d=j} a_{d}^{*}+\beta X_{j t}^{*}+\sum_{l=1}^{D-1} \sum_{d=1}^{D-1} \nVdash_{d=j} b_{d l}^{*} Y_{l t}^{*}+\sum_{d=1}^{D-1} \nVdash_{d=j} c_{d}^{*} Z_{t}+\epsilon_{j t}^{*}
$$

with $\nVdash_{d=j}=1$ if $d=j$ and 0 otherwise, and $\epsilon_{j t}^{*} \sim \mathcal{N}\left(0, \sigma^{2}\right) \quad \forall j=1, \ldots, D-1, \forall t=1, \ldots, T$. However, the induced constant variance of transformed error terms across coordinates, as in Model A, is questionable. Note that Model B can also be estimated using dummy variables as in equation (8) leading to the same estimated coefficients but not to the same standard errors than in equation (6) because of the assumption on error terms. 
Table 1: Two kinds of models for compositional dependent and explanatory variables

\begin{tabular}{|c|c|c|}
\hline & Model A & Model B \\
\hline In compositions & $\mathbf{S}_{t}=\mathbf{a} \oplus b \odot \mathbf{X}_{t} \oplus Z_{t} \odot \mathbf{c} \oplus \epsilon$ & $\mathbf{S}_{t}=\mathbf{a} \oplus \mathbf{B} \odot \mathbf{X}_{t} \oplus Z_{t} \odot \mathbf{c} \oplus \epsilon$ \\
\hline In attraction form & $S_{j t}=\frac{a_{j} X_{j t}^{b} c_{j}^{Z_{t}} \epsilon_{j t}}{\sum_{m=1}^{D} a_{m} X_{m t}^{b} C_{m}^{Z_{t}} \epsilon_{m t}}$ & $S_{j t}=\frac{a_{j} \prod_{l=1}^{D} X_{l t}^{b_{j l}} c_{j}^{Z_{t}} \epsilon_{j t}}{\sum_{m=1}^{D} a_{m} \prod_{l=1}^{D} X_{l t}^{b_{m l}} c_{m}^{Z_{t}} \epsilon_{m t}}$ \\
\hline In coordinates & $\mathbf{S}_{t}^{*}=\mathbf{a}^{*}+\mathbf{X}_{t}^{*} b+\mathbf{c}^{*} Z_{t}+\epsilon_{t}^{*}$ & $\mathbf{S}_{t}^{*}=\mathbf{a}^{*}+\mathbf{X}_{t}^{*} \mathbf{B}_{\mathbf{k}}^{*}+\mathbf{c}^{*} Z_{t}+\epsilon_{t}^{*}$ \\
\hline Component-specific & $\begin{array}{c}\text { with } \epsilon_{j t}^{*} \sim \mathcal{N}\left(0, \sigma^{2}\right) \quad \forall j, \forall t \\
\text { No }\end{array}$ & $\begin{array}{c}\text { with } \epsilon_{j t}^{*} \sim \mathcal{N}\left(0, \sigma_{j}^{2}\right) \quad \forall t \\
\text { Yes }\end{array}$ \\
\hline Cross-effects for $X$ & No & Yes \\
\hline Dimension & $D$ for $\mathbf{S}$ and $\mathbf{X}$ & $D_{S}$ for $\mathbf{S} ; D_{X}$ for $\mathbf{X}$ \\
\hline Nb. parameters & $(D-1)\left(1+K_{Z}\right)+K_{X}$ & $\left(D_{S}-1\right)\left(1+K_{Z}+\sum_{k=1}^{K_{X}}\left(D_{k}-1\right)\right)$ \\
\hline \multicolumn{3}{|c|}{$\begin{array}{l}\mathbf{X}_{t} \text { : compositional explanatory variable; } Z_{t} \text { : classical explanatory variable. } \\
D_{S}: \text { number of components of } \mathbf{S} ; D_{X} \text { or } D_{k}: \text { number of components of } \mathbf{X}_{\mathbf{k}} \text {. } \\
\mathbf{S}, \mathbf{a}, \mathbf{b}, \mathbf{X}, \epsilon \in \mathcal{S}^{D} ; b, X \in \mathbb{R} ; \mathbf{B} \in \mathbb{R}^{D_{S} \times D_{X}} ; \mathbf{S}^{*}, \mathbf{a}^{*}, \mathbf{b}^{*}, \mathbf{B}_{\mathbf{k}}^{*}, \mathbf{X}^{*}, \epsilon^{*}: \text { ILR coordinates. } \\
\epsilon \text { : normal in the simplex distributed error terms } ; \epsilon^{*}: \text { normal distributed error terms. }\end{array}$} \\
\hline
\end{tabular}

As Model A and Model AB are constrained versions of Model B, a model selection can be done using a Fisher test. This requires that the distributions of the error terms of these models are supposed to be Gaussian.

We consider testing the following null hypothesis : $H_{0}: b_{j, j}^{*}=b^{*} \quad \forall j \quad$ and $\quad b_{j, l}^{*}=0 \quad \forall j \neq$ $l$. The associate test statistic is:

$$
\begin{aligned}
F & =\frac{S S E_{0}-S S E_{1}}{S S E_{1}} \times \frac{N-K}{p} \sim \mathcal{F}(p, N-K) \text { under } H_{0} \\
& =\frac{S S E_{0}-S S E_{1}}{S S E_{1}} \times \frac{(D-1)\left[T-K(D-1)-K_{Z}-1\right]}{p}
\end{aligned}
$$

with $S S E_{0}$ and $S S E_{1}$ the sum of squared errors of the constraint and non-constraint models, $T$ the number of observations, $N=(D-1) T$ the number of observed coordinates, $K$ the number of compositional explanatory variables, $K_{Z}$ the number of non-compositional explanatory variables, and $p$ the number of constraints.

\section{Interpretation of compositional models}

As the estimation of compositional models is performed in the coordinate space, the interpretation of the fitted parameters is difficult because parameters are linked to the log-ratio transformation of shares, not directly to the shares. It is possible to derive the coefficients in the simplex associated to shares using the inverse transformation, but their interpretation is not straightforward either.

We are going to show that relative impacts, like elasticities or odds ratios, are more natural (as is the case of the classical logistic model) than marginal effects, to interpret impacts on shares.

Table 2 compares the different measures of impact assessment of explanatory variables (compositional and non-compositional) in Model A and Model B, which are detailed below. Note that it is not possible to measure the impact of the share of $X_{l t}$, but only of the corresponding volume of $\check{X}_{l t}$. Indeed, a share cannot increase ceteris paribus because it implies a change in 
other shares. However, we can consider a change in the volume of $\check{X}_{l t}$, with all other volumes $\check{X}_{m t}, m \neq l$ fixed.

\subsection{Marginal effect of a component}

In classical linear models, coefficients are usually interpreted in terms of marginal effects: if the explanatory variable increases by one, then the dependent variable increases by the value of the coefficient. In the case of compositional models, we prove in this paper that it is possible to compute marginal effects, but it is not straightforward. The marginal effect of the component $\check{X}_{l t}$ (in volume) on the dependent share $S_{j t}$ is defined as:

$$
m e\left(\mathbb{E}^{\oplus} S_{j t}, \check{X}_{l t}\right)=\frac{\partial \mathbb{E}^{\oplus} S_{j t}}{\partial \check{X}_{l t}}
$$

where $\mathbb{E}^{\oplus} S_{j t}$ is the "expected value in the simplex" of $S_{j t}$ (see Morais et al. (2017)), such that $\mathbb{E}^{\oplus} S_{j t}=\frac{a_{j} X_{j t}^{b} c_{j}^{Z_{t}}}{\sum_{m=1}^{D} a_{m} X_{j t}^{b} c_{m}^{Z_{t}}}$ for Model A and $\mathbb{E}^{\oplus} S_{j t}=\frac{a_{j} \prod_{l=1}^{D} X_{l t}^{b_{j l}} c_{j}^{Z_{t}}}{\sum_{m=1}^{D} a_{m} \prod_{l=1}^{D} X_{l t}^{b_{m} l} c_{m}^{Z_{t}}}$ for Model B.

For Model B, we show that marginal effects can be computed as follows:

$$
m e\left(\mathbb{E}^{\oplus} S_{j t}, \check{X}_{l t}\right)=\frac{\partial \mathbb{E}^{\oplus} S_{j t}}{\partial \log \mathbb{E}^{\oplus} S_{j t}} \frac{\partial \log \mathbb{E}^{\oplus} S_{j t}}{\partial \log \check{X}_{l t}} \frac{\partial \log \check{X}_{l t}}{\partial \check{X}_{l t}}=\left(b_{j l}-\sum_{m=1}^{D} S_{m t} b_{m l}\right) \frac{\mathbb{E}^{\oplus} S_{j t}}{\check{X}_{l t}}
$$

If $M E_{D_{S}, D_{X}}$ is the matrix containing all marginal effects, we then have:

$$
M E\left(\mathbb{E}^{\oplus} \mathbf{S}_{t}, \check{\mathbf{X}}_{t}\right)=\left[\mathbf{S}_{\mathbf{j t}}\right] \odot \mathbf{W}_{t} \mathbf{B} \odot\left[\frac{\mathbf{1}}{\check{\mathbf{X}}_{\mathbf{l t}}}\right]=\left[\mathbf{S}_{\mathbf{j t}}\right] \odot \mathbf{W}_{t} \mathbf{V} \mathbf{B}^{*} \mathbf{V}^{\prime} \odot\left[\frac{\mathbf{1}}{\check{\mathbf{X}}_{\mathbf{l t}}}\right]
$$

where $\odot$ denotes here the Hadamard product (term by term product) ${ }^{4},\left[\mathbf{S}_{\mathbf{j} \mathbf{t}}\right]$ is a $D_{S} \times D_{S}$ matrix with $S_{j t}$ on the $j^{\text {th }}$ row, $\left[\frac{1}{\dot{\mathbf{X}}_{\mathrm{lt}}}\right]$ is a $D_{X} \times D_{X}$ matrix with $\check{X}_{l t}$ on the $l^{\text {th }}$ column, $\mathbf{B}^{*}$ and $\mathbf{B}$ denote the parameters in the transformed space and in the simplex, and $\mathbf{W}_{t}$ is a $D_{S} \times D_{S}$ matrix composed of diagonal terms equal to $1-\mathbb{E}^{\oplus} S_{j}$ and non-diagonal terms in column $j$ equal to $-\mathbb{E}^{\oplus} S_{j}$. Similar results can be found for Model A in Table 2, where $\mathbf{B}$ is replaced by $b$.

This marginal effect matrix can also be computed using ILR coordinates and Jacobian matrices instead of using the attraction form of the model (see Appendix A.2 for details).

\subsection{Elasticity of a dependent share relative to a component}

The marginal effect $m e\left(\mathbb{E}^{\oplus} S_{j t}, \check{X}_{l t}\right)$ depends on all shares $S_{m t}$ and on volumes $\check{X}_{l t}$. We can see in our application that it can vary a lot across observations, and therefore it is not a good measure to summarize the impact of a component $\check{X}_{l t}$ on a share $S_{j t}$. We are going to show that elasticities are more natural to interpret compositional models.

The first elasticity we may want to compute is the elasticity of the share $S_{j t}$ relative to the volume of $\check{X}_{l t}$. It corresponds to the relative variation of $S_{j t}$ induced by a relative variation of $1 \%$ of the volume $\check{X}_{l t}$ (keeping all other volumes constant) or alternatively a relative variation of $1 \%$ of the share $X_{l t}$ (holding constant the ratios $\frac{X_{i t}}{X_{j t}}$ of the remaining components).

$$
e_{j l t}=e\left(\mathbb{E}^{\oplus} S_{j t}, \check{X}_{l t}\right)=\frac{\frac{\partial \mathbb{E}^{\oplus} S_{j t}}{\mathbb{E}^{\oplus} S_{j t}}}{\frac{\partial \check{X}_{l t}}{\check{X}_{l t}}}=\frac{\partial \log \mathbb{E}^{\oplus} S_{j t}}{\partial \log \check{X}_{l t}}
$$

Since both variables (dependent and independent) are compositions, we should consider the notion of derivative of a simplex valued function with respect to a compositional variable.

\footnotetext{
${ }^{4}$ Note that $\odot$ in bold denotes the Hadamard product whereas $\odot$ denotes the power transformation.
} 
Egozcue et al. (in Pawlowsky-Glahn and Buccianti (2011), chapter 12) treat the case of the derivative of a simplex-valued function of a real variable and Barcelo-Vidal et al. (in Pawlowsky-Glahn and Buccianti (2011), chapter 13) the case of the derivative of a vector valued function of a compositional variable. Combining the two notions, let us denote by $\frac{\partial^{\oplus} \mathbf{h}}{\partial \oplus X_{l}}$ the directional $\mathcal{C}$ derivatives of a function $\mathbf{h}$ from the simplex $\mathcal{S}^{D_{X}}$ to the simplex $\mathcal{S}^{D_{S}}$. Using a result (see appendix A.3) linking the directional $\mathcal{C}$ derivatives of the function $\mathbf{h}$ of shares with the semi-log derivatives of the corresponding function of volumes, we can then derive the relationship between the directional C-derivatives of the share vector $\mathbf{S}_{t}$ with respect to the shares $X_{l t}$ and the above elasticities as follows:

$$
e_{l t}^{\oplus}=\frac{\partial^{\oplus} \mathbb{E}^{\oplus} \mathbf{S}_{t}}{\partial^{\oplus} X_{l t}}=\mathcal{C}\left(\exp \left(\frac{\partial \log \mathbb{E}^{\oplus} \mathbf{S}_{t}}{\partial \log \check{X}_{l t}}\right)\right)^{\prime}=\mathcal{C}\left(\exp \left(e_{1 l t}\right), \ldots, \exp \left(e_{D l t}\right)\right)^{\prime}
$$

The elasticities $e_{j l t}$ from (12) are easy to compute from the attraction form of $\mathbb{E}^{\oplus} S_{j t}$, in a similar way than marginal effects (see equation (10)). They can also be expressed in matrix form $E\left(\mathbb{E}^{\oplus} \mathbf{S}_{t}, \check{\mathbf{X}}_{t}\right)$ (results are in Table 2). The relationship between marginal effects and elasticities is as follows:

$$
M E\left(\mathbb{E}^{\oplus} \mathbf{S}_{t}, \check{\mathbf{X}}_{t}\right)=\left[\mathbf{S}_{\mathbf{j} \mathbf{t}}\right] \odot E\left(\mathbb{E}^{\oplus} \mathbf{S}_{t}, \check{\mathbf{X}}_{t}\right) \odot\left[\mathbf{1} / \check{\mathbf{X}}_{\mathbf{l t}}\right]
$$

where $\odot$ denotes the Hadamard product.

These elasticities allow to isolate the impact of one $\check{X}$ 's component on one S's component which is very useful. This impact is understood as the impact of a relative variation of the volume (resp: the share) keeping all other volumes constant (resp: holding constant the ratios of the remaining components). Compared to marginal effects, the $e\left(\mathbb{E}^{\oplus} S_{j t}, \check{X}_{l t}\right)$ still depend on observations through the shares $S_{m t}$, but do not depend any more on the volumes $\check{X}_{l t}$. Then, if shares are not varying too much, as it is the case in our example (see Section 4), they can be a good measure of impact.

As for marginal effects, the elasticity matrix can also be computed from ILR coordinates (see Appendix A.2 for details).

Let us now consider making a first order Taylor approximation of the vector of shares for a small relative change in component $l$. For a small $\delta=\frac{\Delta \check{X}_{l t}}{\check{X}_{l t}}$, we could write this first approximation of the share:

$$
S_{j t}^{\prime}=S_{j t}\left(1+\delta e_{j l t}\right)
$$

and it is easy to see that $\mathbf{S}_{t}^{\prime}=\left(S_{1 t}^{\prime}, \ldots, S_{D t}^{\prime}\right)^{\prime}$ does belong to the simplex (they are summing up to one because $\sum_{m=1}^{D} \mathbb{E}^{\oplus} S_{m t} e_{j l t}=0$ and $\sum_{m=1}^{D} \mathbb{E}^{\oplus} S_{m t}=1$, see proof in the appendix A.4). Another first order Taylor approximation of the vector of shares denoted $\mathbf{S}_{t}^{\prime \prime}$ is ${ }^{5}$ :

$$
\mathbf{S}_{t}^{\prime \prime}=\mathbf{S}_{t} \oplus \delta \odot e_{l t}^{\oplus}=\mathcal{C}\left(S_{1 t} \exp \left(\delta e_{1 l t}\right), \ldots, S_{D t} \exp \left(\delta e_{D l t}\right)\right)^{\prime}
$$

Note that when $\delta \rightarrow 0$, since $\exp \left(\delta e_{j l t}\right) \simeq 1+\delta e_{j l t}$ these two approximations are equivalent at first order, although the first one is simpler as it uses the Euclidean geometry instead of the Aitchison geometry:

$$
\mathbf{S}_{t}^{\prime \prime} \simeq \mathcal{C}\left(S_{1 t}\left(1+\delta e_{1 l t}\right), \ldots, S_{D t}\left(1+\delta e_{D l t}\right)\right)^{\prime}=\mathcal{C}\left(S_{1 t}^{\prime}, \ldots, S_{D t}^{\prime}\right)^{\prime}=\left(S_{1 t}^{\prime}, \ldots, S_{D t}^{\prime}\right)^{\prime}
$$

\subsection{Elasticity and odds ratio of a ratio of dependent shares relative to a component}

In order to avoid being observation dependent, other measures can be computed for interpreting Models A and B. However, they are concerning ratios of shares, not directly a single share. Then, they can be complicated to interpret in practical cases.

\footnotetext{
${ }^{5}$ See equation (12.13), p.168, in Pawlowsky-Glahn and Buccianti (2011).
} 
Elasticity of a ratio of dependent shares As compositional data analysis is based on a log-ratio approach, elasticities of ratios are easy to compute. We can be interested in the elasticity of a ratio of shares (or volumes) $\mathbb{E}^{\oplus} S_{j t} / \mathbb{E}^{\oplus} S_{j^{\prime} t}$ relative to an infinitesimal change in the volume of $\check{X}_{l t}$.

$$
e\left(\mathbb{E}^{\oplus} S_{j t} / \mathbb{E}^{\oplus} S_{j^{\prime} t}, \check{X}_{l t}\right)=\frac{\partial \log \left(\mathbb{E}^{\oplus} S_{j t} / \mathbb{E}^{\oplus} S_{j^{\prime} t}\right)}{\partial \log \check{X}_{l t}}
$$

We see in Table 2 that the result is constant across observations because it only depends on parameters. Note here that Model A respects the IIA (Independence from Irrelevant Alternatives) property, meaning that the ratio of two shares $\mathbb{E}^{\oplus} S_{j t} / \mathbb{E}^{\oplus} S_{j^{\prime} t}$ only depends on the corresponding components $j$ and $j^{\prime}$ of $\check{\mathbf{X}}$. Then, $e\left(\mathbb{E}^{\oplus} S_{j t} / \mathbb{E}^{\oplus} S_{j^{\prime} t}, \breve{X}_{l t}\right)=0$ if $l \neq j, j^{\prime}$. Moreover, the elasticity of the ratio between the share $j$ and the share $j^{\prime}$ relative to a change in $\check{X}_{j t}$ is the same for all considered shares $j^{\prime}$. This is a lack of flexibility of Model A, because it implies that an increase of $\check{X}_{j t}$ will reduce proportionally all other shares. Model B does not satisfy the IIA property, and then this model is able to take into account possible synergies between brands.

Odds ratio of a ratio of dependent shares Another type of interpretation which can be used for shares is the odds ratio. The advantage of this measure is that it is a measure of impact of a discrete change, as opposed to infinitesimal change, of $\check{X}_{l}$ ( $\check{X}_{l}$ is increased by $\Delta \times 100 \%$ between situations $t=t 1$ and $t=t 2)$ on the ratio $\mathbb{E}^{\oplus} S_{j t} / \mathbb{E}^{\oplus} S_{j^{\prime} t}$. The odds ratio for a couple of shares $\mathbb{E}^{\oplus} S_{j t} / \mathbb{E}^{\oplus} S_{j^{\prime} t}$ relative to $\check{X}_{l t}$ is given by:

$$
O R\left(\mathbb{E}^{\oplus} S_{j t} / \mathbb{E}^{\oplus} S_{j^{\prime} t}, \check{X}_{l t}, \Delta\right)=\frac{\left(\mathbb{E}^{\oplus} S_{j, t 2} / \mathbb{E}^{\oplus} S_{j^{\prime}, t 2}\right) \mid \check{X}_{l, t 2}}{\left(\mathbb{E}^{\oplus} S_{j, t 1} / \mathbb{E}^{\oplus} S_{j^{\prime}, t 1}\right) \mid \check{X}_{l, t 1}}
$$

where $\check{X}_{l, t 2}=(1+\Delta) \check{X}_{l, t 1}$ and $\Delta \geq 0$.

Remark: $e\left(\mathbb{E}^{\oplus} S_{j t} / \mathbb{E}^{\oplus} S_{j^{\prime} t}, \check{X}_{l t}\right)$ and $O R\left(\mathbb{E}^{\oplus} S_{j t} / \mathbb{E}^{\oplus} S_{j^{\prime} t}, \check{X}_{l t}, \Delta\right)$ are more or less measuring the same thing differently, if $\Delta$ is small:

$$
\begin{aligned}
e\left(\mathbb{E}^{\oplus} S_{j t} / \mathbb{E}^{\oplus} S_{j^{\prime} t}, \check{X}_{l t}\right) & \simeq \frac{\left(\mathbb{E}^{\oplus} S_{j t 2} / \mathbb{E}^{\oplus} S_{j^{\prime} t 2}\right)-\left(\mathbb{E}^{\oplus} S_{j t 1} / \mathbb{E}^{\oplus} S_{j^{\prime} t 1}\right)}{\left(\mathbb{E}^{\oplus} S_{j t 1} / \mathbb{E}^{\oplus} S_{j^{\prime} t 1}\right)} / \frac{\check{X}_{l t 2}-\check{X}_{l t 1}}{\check{X}_{l t 1}} \\
& \simeq \frac{O R\left(\mathbb{E}^{\oplus} S_{j t} / \mathbb{E}^{\oplus} S_{j^{\prime} t}, \check{X}_{l t}, \Delta\right)-1}{\left(\check{X}_{l t 2}-\check{X}_{l t 1}\right) /\left(\check{X}_{l t 1}\right)}
\end{aligned}
$$

\subsection{Elasticity of a particular ratio of dependent shares relative to a partic- ular ratio of components}

Usually, compositional models are interpreted directly on coordinates. Thus, it is advised to choose an appropriate ILR transformation in order to have ILR coordinates which make sense for the considered application, using sequential binary partition e.g. pivot coordinates (Fišerová and Hron (2011)) as a special case of balances (see Hron, Filzmoser, and Thompson (2012)). But, previously the interpretation was made in terms of marginal effects on ILR coordinates, that is marginal effects on a particular log-ratio of shares. We show here that we can go a step further and make an interpretation in terms of elasticity for the ratio of shares directly.

Chen et al. (2017) interpret in the case of Model B the impact of the ratio $X_{l} / g\left(X_{-l}\right)=$ $\check{X}_{l} / g\left(\check{X}_{-l}\right)$ on the ratio $\mathbb{E}^{\oplus} S_{j} / g\left(\mathbb{E}^{\oplus} S_{-j}\right)=\mathbb{E}^{\oplus} \check{S}_{j} / g\left(\mathbb{E}^{\oplus} \check{S}_{-j}\right.$ ) (ratios on shares or volumes are equivalent), which is the ratio of a particular share (or volume) $S_{j}$ over the geometric average of other shares (or volumes). The adapted pivot coordinates are the following:

$$
i \operatorname{lr}(\mathbf{X})_{i}=\sqrt{\frac{D-i}{D-i+1}} \log \frac{x_{i}}{\left(\prod_{j=1+i}^{D} x_{j}\right)^{1 /(D-i)}}, \quad i=1, \ldots, D-1
$$


which is equivalent to the following balance matrix in the case of $D=4$ :

$$
V_{I L R, 4}=\left[\begin{array}{ccc}
\sqrt{3 / 4} & 0 & 0 \\
-\sqrt{1 / 12} & \sqrt{2 / 3} & 0 \\
-\sqrt{1 / 12} & -\sqrt{1 / 6} & \sqrt{1 / 2} \\
-\sqrt{1 / 12} & -\sqrt{1 / 6} & -\sqrt{1 / 2}
\end{array}\right]
$$

With this transformation, the first expected coordinate of $\mathbf{S}$ in Model A, is equal to:

$$
\mathbb{E} i \operatorname{il}(\mathbf{S})_{1}=\sqrt{\frac{D-1}{D}} \log \frac{\mathbb{E}^{\oplus} S_{1 t}}{g\left(\mathbb{E}^{\oplus} S_{-1 t}\right)}=a_{1}^{*}+b^{*} \sqrt{\frac{D-1}{D}} \log \frac{\check{X}_{1 t}}{g\left(\check{X}_{-1 t}\right)}+c_{1}^{*} Z_{t}
$$

In Model $\mathrm{B}$, the first expected coordinate of $\mathbf{S}$ is equal to:

$$
\begin{aligned}
& \mathbb{E} i \operatorname{li}(\mathbf{S})_{1}=\sqrt{\frac{D_{S}-1}{D_{S}}} \log \frac{\mathbb{E}^{\oplus} S_{1 t}}{g\left(\mathbb{E}^{\oplus} S_{-1 t}\right)}=a_{1}^{*}+b_{11}^{*(j, l)} \sqrt{\frac{D_{X}-1}{D_{X}}} \log \frac{\check{X}_{1 t}}{g\left(\check{X}_{-1 t}\right)} \\
& +b_{12}^{*(j, l)} \sqrt{\frac{D_{X}-2}{D_{X}-1}} \log \frac{\check{X}_{2 t}}{g\left(\check{X}_{-1-2 t}\right)}+\ldots
\end{aligned}
$$

In order to interpret their model, Chen et al. (2017) compute the marginal effect of $\operatorname{lr}(X)_{1}^{(l)}$ on $\operatorname{ilr}(S)_{1}^{(j)}$ :

$$
m e\left(\mathbb{E} i l r(S)_{1}^{(j)}, i \operatorname{lir}(\check{X})_{1}^{(l)}\right)=\frac{\partial \sqrt{\frac{D_{S}-1}{D_{S}}} \log \left(\mathbb{E}^{\oplus} S_{j t} / g\left(\mathbb{E}^{\oplus} S_{-j t}\right)\right)}{\partial \sqrt{\frac{D_{X}-1}{D_{X}}} \log \left(\check{X}_{l t} / g\left(\check{X}_{-l t}\right)\right)}=b_{11}^{*(j, l)}
$$

such that an increase of one unit of $i \operatorname{lr}(\check{X})_{1}^{(l)}$ implies an increase of $b_{11}^{*(j, l)}$ units of $\mathbb{E} i \operatorname{lr}(S)_{1}^{(j) 6}$. Note that this is only true if $\sqrt{\frac{D_{X}-1}{D_{X}}} \log \left(X_{l t} / g\left(X_{-l t}\right)\right)$ moves because $\check{X}_{1 t}$ moves while other $\check{X}_{j t}$ remain constant. Otherwise, other ILR coordinates in the right part of the equation are moving and the marginal effect should take it into account. However, for Model A, we do not have this problem because other ILR coordinates of $\mathbf{X}$ are not used.

We show that this is equivalent to compute the following elasticity (multiplying by a factor if $\left.D_{S} \neq D_{X}\right)$ :

$$
e\left(\frac{\mathbb{E}^{\oplus} S_{j t}}{g\left(\mathbb{E}^{\oplus} S_{-j t}\right)}, \check{X}_{l t}\right)=\frac{\partial \log \left(\mathbb{E}^{\oplus} S_{j t} / g\left(\mathbb{E}^{\oplus} S_{-j t}\right)\right)}{\partial \log \check{X}_{l t}}=\sqrt{\frac{\left(D_{X}-1\right) / D_{X}}{\left(D_{S}-1\right) / D_{S}}} b_{11}^{*(j, l)}
$$

Thus, instead of saying that when $i \operatorname{lr}(\check{X})_{1}^{(l)}$ increases by 1 unit, $\mathbb{E} i \operatorname{lr}(S)_{1}^{(j)}$ increases by $b_{11}^{*(j, l)}$ units, one can say that when $\check{X}_{l t}$ increases by $1 \%, \mathbb{E}^{\oplus} S_{j t} / g\left(\mathbb{E}^{\oplus} S_{-j t}\right)$ increases by $b_{11}^{*(j, l)} \%$ (in the case where $D_{S}=D_{X}$ ). Note that this $b_{11}^{*(j, l)}$ will be different for each permutation (i.e. each couple $j, l$ ). Chen et al. (2017) show how one can determine in one step the first coefficient of $B^{*(j, l)}$, the $b_{11}^{*(j, l)}$ which is used to compute the above elasticity, for all possible permutations without fitting several times the model.

\subsection{Elasticities and odds ratios relative to a non-compositional variable}

The same kind of interpretations can be done for non-compositional variables $Z$, as presented in Table 2, except for the elasticity including the geometrical mean.

Indeed, this would allow to measure the marginal effect (not the elasticity) of $Z_{t}$ over

$$
\sqrt{\frac{D_{S}-1}{D_{S}}} \log \frac{S_{1 t}}{g\left(S_{-1 t}\right)} .
$$

\footnotetext{
${ }^{6} \operatorname{ilr}(S)_{1}^{(j)}$ denotes the first ILR coordinate of $\mathbf{S}$ where $S_{j}$ is in the first position; $\operatorname{ilr}(\check{X})_{1}^{(l)}$ denotes the first ILR coordinate of $\check{\boldsymbol{X}}$ where $\check{X}_{l}$ is in the first position.
} 
Table 2: Measures of impact assessment for Model A and Model B

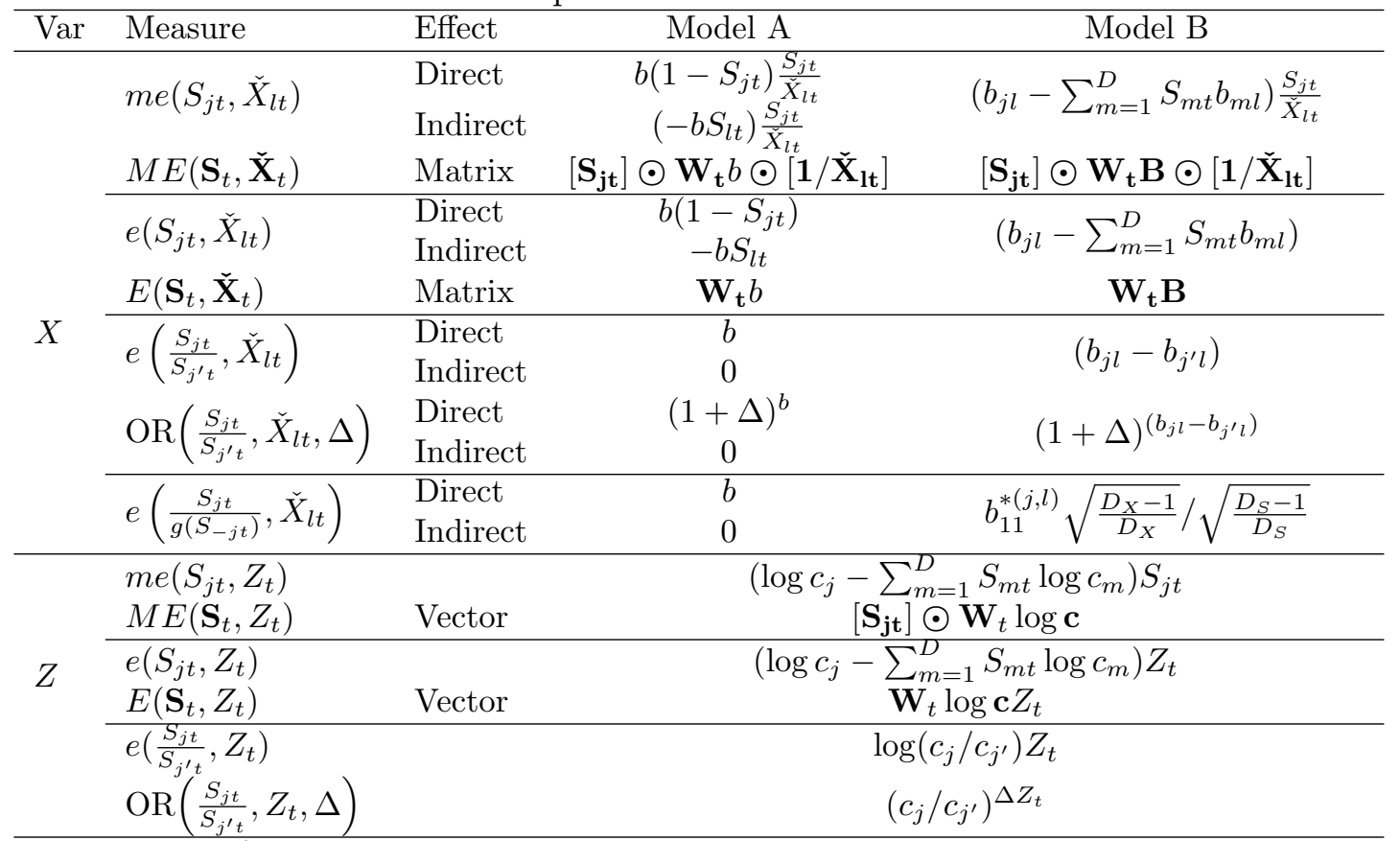

In this table, $\mathbb{E}^{\oplus} S_{j t}$ is denoted by $S_{j t}$ to shorten notations, and $\odot$ denotes the Hadamard product. Moreover, these measures are estimated using observed shares $S_{j t}$ in practice, not fitted shares.

Direct effect when $l=j$; indirect effect when $l \neq j$.

$\mathbf{W}_{\mathbf{t}}$ contains $1-S_{i t}$ on the diagonal and $-S_{i t}$ otherwise.

This marginal effect would be equal to $c_{1}^{*}$ for Model A and Model B, but this kind of interpretation is not useful to understand the impact of $Z$ on the final shares. Thus, we do not show this measure in Table 2.

Note that in practice, elasticities and other measures depending on $\mathbb{E}^{\oplus} S_{j t}$ are estimated using the observed shares $S_{j t}$, not the fitted shares $\widehat{S_{j t}}$.

\section{Impact of media investments on brands market-shares}

In Europe, the automobile market is usually segmented in 5 segments, from A to E, according to the size of the vehicle chassis. Within each segment, one can suppose that consumers intending to buy new cars make their choice between brands ${ }^{7}$ according to the price and the "image" of the brand. The image of the brand is supposed to reflect the notion of quality and reliability of the brand. Car manufacturers spend millions of euros in media investments to enhance their image, giving rise to the following question: do the media investments have an impact on brands market-shares ${ }^{8}$ ?

In order to answer this question in the present paper, we model brands market-shares of the B segment of the French automobile market ${ }^{9}$ as a function of brand media investments (in $\mathrm{TV}$, radio, press, outdoor, internet and cinema), of brand average catalogue price and of a scrapping incentive dummy variable. In a further work, we consider modeling other segments, and differentiate media investments according to channels.

\footnotetext{
${ }^{7}$ Inside a segment, a brand generally supplies only one main vehicle. Thus, we can consider that the alternatives for a consumer inside a particular segment coincide with the available brands in this segment.

${ }^{8}$ We decide to ask the question in terms of market-shares instead of in terms of sales volumes because one can suppose that at time $t$, brands have to share a market for which the size is mainly determined by the demand.

${ }^{9}$ The B segment is the most important segment in terms of sales in France (around $40 \%$ of new passenger car sales).
} 
In this paper, three brands are highlighted (Renault, Peugeot, Citroen, the leaders of the B segment) while other brands of the B segment are aggregated in a category "Others" (see Figure 1). The media investments are the sum of TV, radio, press, outdoor, internet and cinema investments in euros by brands for their vehicles in the B segment (see Figure 1). They do not include advertising budget for the brand itself. Actually we use the media investments of one, two and three months before the purchase time (at time $t-1, t-2, t-3$ ) as explanatory variables. The average brand price (average of catalogue prices weighted by corresponding sales at the vehicle level) is also used as an explanatory variable (see Figure 1). It does not include potential promotions made in the car dealership at the time of purchase. Even if they do not vary a lot across time, prices are used to position brands within the segment. We also control for scrapping incentive periods. The corresponding dummy variable is a "classical" variable (not compositional) and varies across time only, not across brands.

Model A and Model B can be considered in this framework: Model A considers that the effect of media investments and price are the same for all brands whereas Model B implies crosseffects and brand-specific impacts of media investments and price on market-shares. As our interest is on the impact of media investments, we also consider the Model AB which contains cross-effects and brand-specific parameters for media investments, but a unique parameter for all brands for the composition of prices.
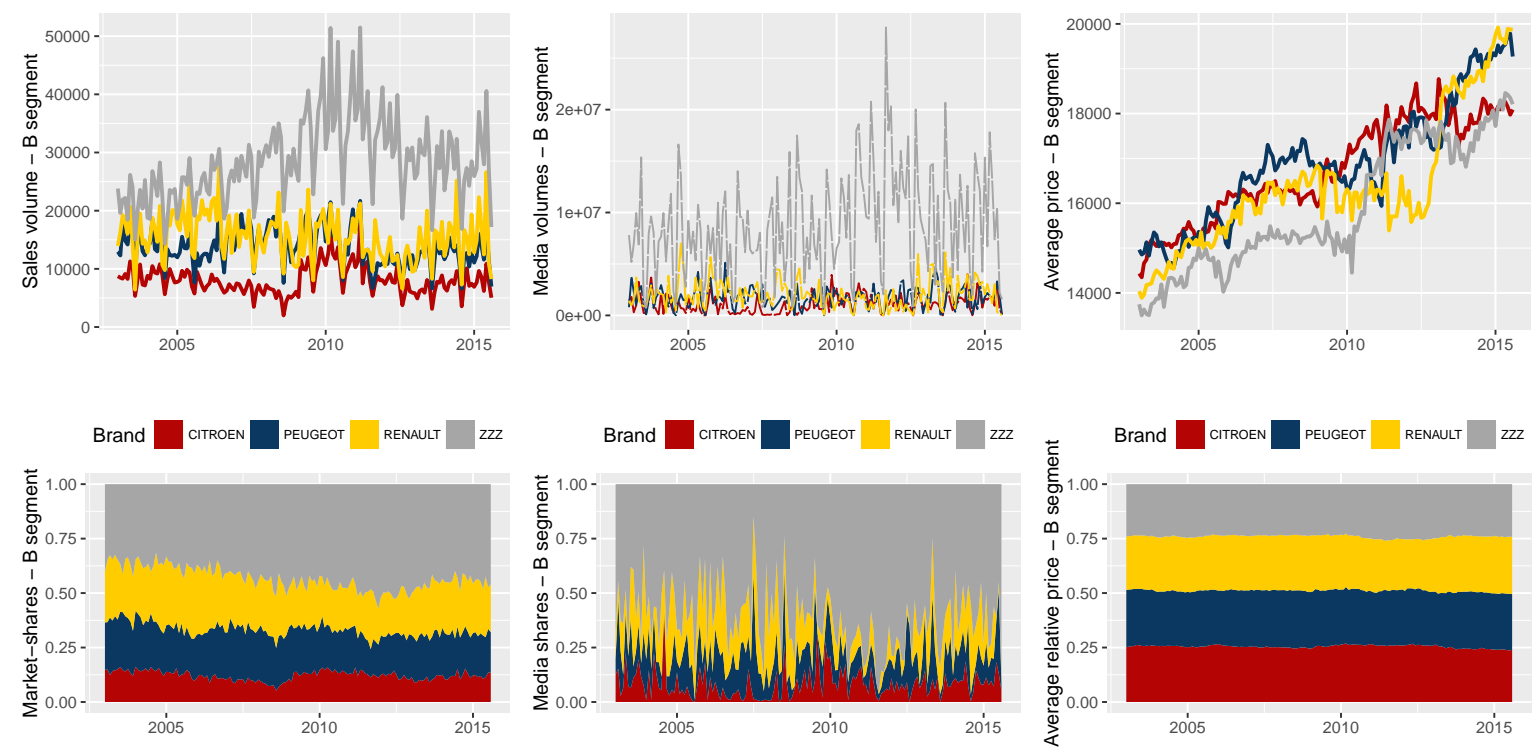

Figure 1: Sales, media and average price of brands, in volume and in share, in the B segment

This section presents the results of this application. We interpret the two models A and B in terms of elasticities and odds ratios of shares, and we compare them in terms of goodnessof-fit measures. The Fisher tests comparing the non-constrained Model B to the constrained Model A and Model AB are also computed. All computations have been done with the $\mathrm{R}$ software.

\subsection{Non brand-specific impact of media investments (Model A)}

Model In the case where it is assumed that brand media investments and brand prices have the same effect for all brands, the following equations correspond to the model in the simplex 
and the attraction formulation of the model:

$$
\begin{aligned}
\mathbf{S}_{t} & =\mathbf{a} \bigoplus_{\tau=1}^{3} b_{\tau} \odot \mathbf{M}_{t-\tau} \oplus b_{P} \odot \mathbf{P}_{t} \oplus S I_{t} \odot \mathbf{c} \oplus \boldsymbol{\varepsilon}_{t} \\
\Leftrightarrow S_{j t} & =\frac{a_{j} \prod_{\tau=1}^{3} M_{t-\tau, j}^{b_{\tau}} P_{t, j}^{b_{P}} c_{j}^{S I} \varepsilon_{j t}}{\sum_{m=1}^{4} a_{m} \prod_{\tau=1}^{3} M_{t-\tau, m}^{b_{\tau}} P_{t, m}^{b_{P}} c_{m}^{S I} \varepsilon_{m t}}
\end{aligned}
$$

where $\mathbf{S}, \mathbf{M}_{\mathbf{t}-\tau}, \mathbf{P} \in \mathcal{S}^{4}$ are the compositions of brand sales, of brand media investments at time $t-1, t-2$ and $t-3$, and of brand prices. $b_{\tau}, b_{P} \in \mathbb{R}$ are the parameters associated to compositional explanatory variables and $\mathbf{c} \in \mathcal{S}^{4}$ is a composition of parameters associated to the dummy variable $S I$ (scrapping incentive).

The model expressed in ILR coordinates is:

$$
\begin{aligned}
\mathbf{S}_{t}^{*} & =\mathbf{a}^{*}+\sum_{\tau=1}^{3} b_{\tau} \mathbf{M}_{t-\tau}^{*}+b_{P} \mathbf{P}_{t}^{*}+\mathbf{c}^{*} S I_{t}+\varepsilon_{t}^{*} \\
\Leftrightarrow S_{j t}^{*} & =a_{j}^{*}+\sum_{\tau=1}^{3} b_{\tau}^{*} M_{j, t-\tau}^{*}+b_{P}^{*} P_{j t}^{*}+c_{j}^{*} S I_{t}+\varepsilon_{j t}^{*} \quad \text { for } j=1,2,3
\end{aligned}
$$

where $\varepsilon^{*}$ is generally supposed to be a Gaussian distributed error term. The balance matrix used for the ILR transformation is the default matrix in the R software with the 'compositions' package:

$$
V_{I L R, 4}=\left[\begin{array}{ccc}
-\sqrt{1 / 2} & -\sqrt{1 / 6} & -\sqrt{1 / 12} \\
\sqrt{1 / 2} & -\sqrt{1 / 6} & -\sqrt{1 / 12} \\
0 & \sqrt{2 / 3} & -\sqrt{1 / 12} \\
0 & 0 & \sqrt{3 / 4}
\end{array}\right]
$$

Results All explanatory variables are significant at $0.1 \%$ according to the analysis of variance (ANOVA). Figure 2 compares observed and fitted shares. It confirms that the model succeeds in fitting the main trends of brands market-shares. However, the model underestimates the market-share of "Others" at the beginning of the period, and overestimates it at

\begin{tabular}{|c|c|c|c|c|}
\hline & Estimate & Std. Error & t value & $\operatorname{Pr}(>|t|)$ \\
\hline$a_{1}^{*}$ & 0.3439 & 0.0151 & 22.84 & $0.0000^{* * *}$ \\
\hline$a_{2}^{*}$ & 0.3363 & 0.0159 & 21.19 & $0.0000^{* * *}$ \\
\hline$a_{3}^{*}$ & 0.6620 & 0.0263 & 25.14 & $0.0000^{* * *}$ \\
\hline$b_{1}$ & 0.0267 & 0.0071 & 3.79 & $0.0002^{* * *}$ \\
\hline$b_{2}$ & 0.0241 & 0.0062 & 3.90 & $0.0001^{* * *}$ \\
\hline$b_{3}$ & 0.0264 & 0.0062 & 4.26 & $0.0000^{* * *}$ \\
\hline$b_{P}$ & 1.2217 & 0.2313 & 5.28 & $0.0000^{* * *}$ \\
\hline$c_{1}^{*}$ & -0.0241 & 0.0338 & -0.71 & 0.4758 \\
\hline$c_{2}^{*}$ & -0.1690 & 0.0334 & -5.05 & $0.0000^{* * *}$ \\
\hline$c_{3}^{*}$ & 0.1292 & 0.0336 & 3.84 & $0.0001^{* * *}$ \\
\hline o param. & 10 & & & \\
\hline
\end{tabular}
the end.

The parameters estimated with the ILR transformed model according to the $V_{I L R, 4}$ matrix are presented in Table 3. The corresponding parameters for the model in the simplex (after

Table 3: Estimated parameters on ILR coordinates - Model A

inverse transformation) are in Table 4. We remark that the coefficient associated to the price is positive, which can be surprising, but price here is correlated with the image of quality of the brand, which is very important for the customer who buys a durable and expensive good like a car. 

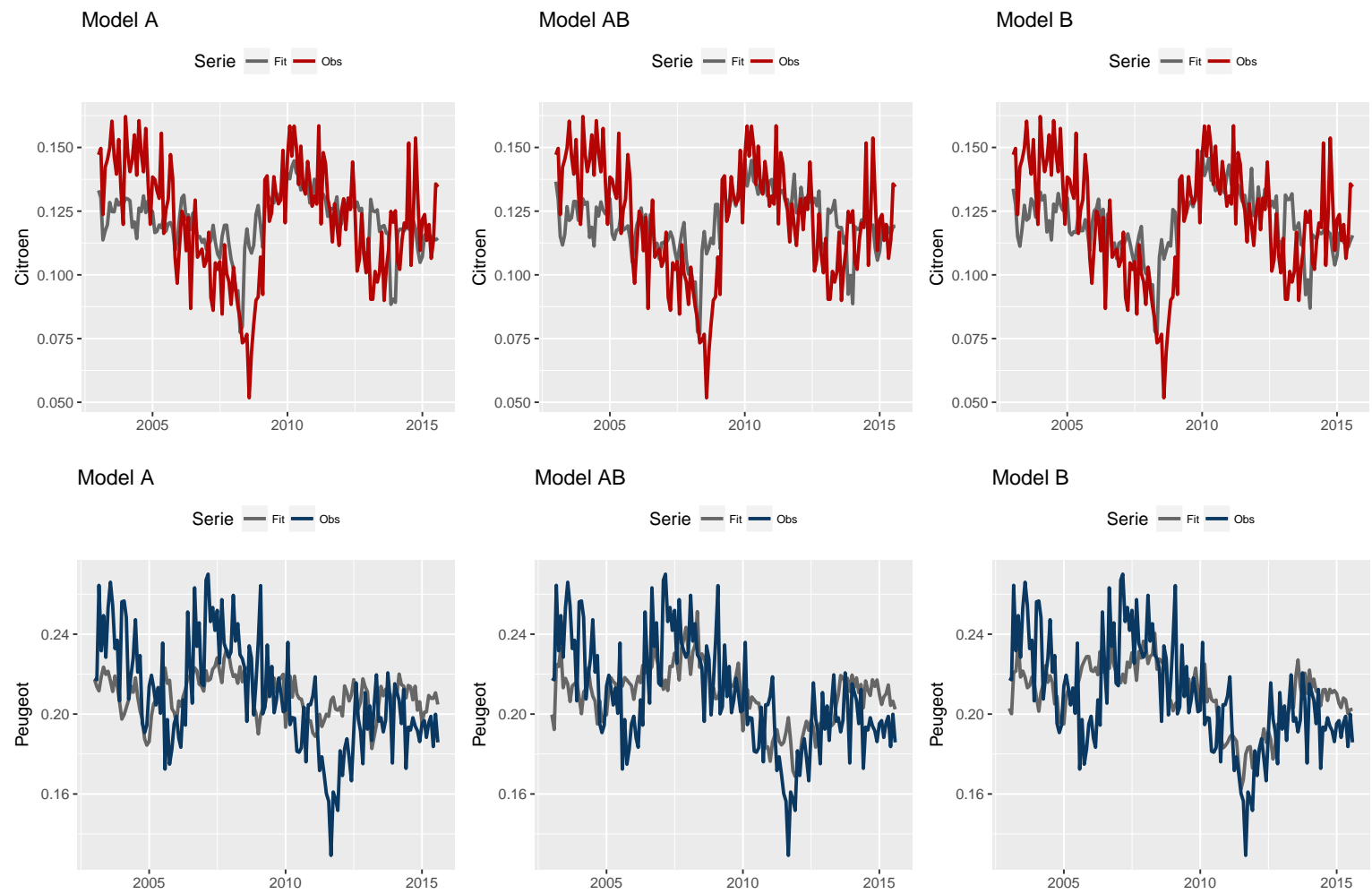

Model B

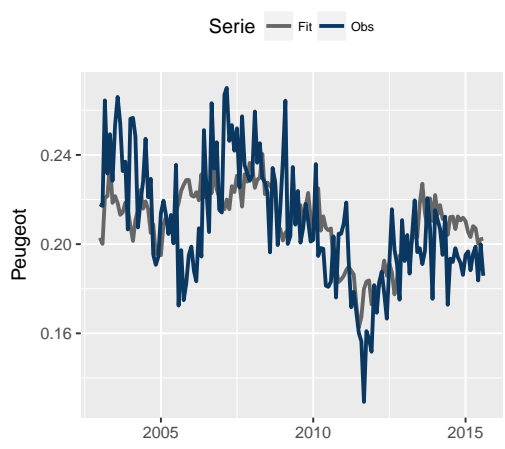

Model A

Model AB

Model B

Serie - Fit - obs

Serie - Fit - obs

Serie - Fit - obs
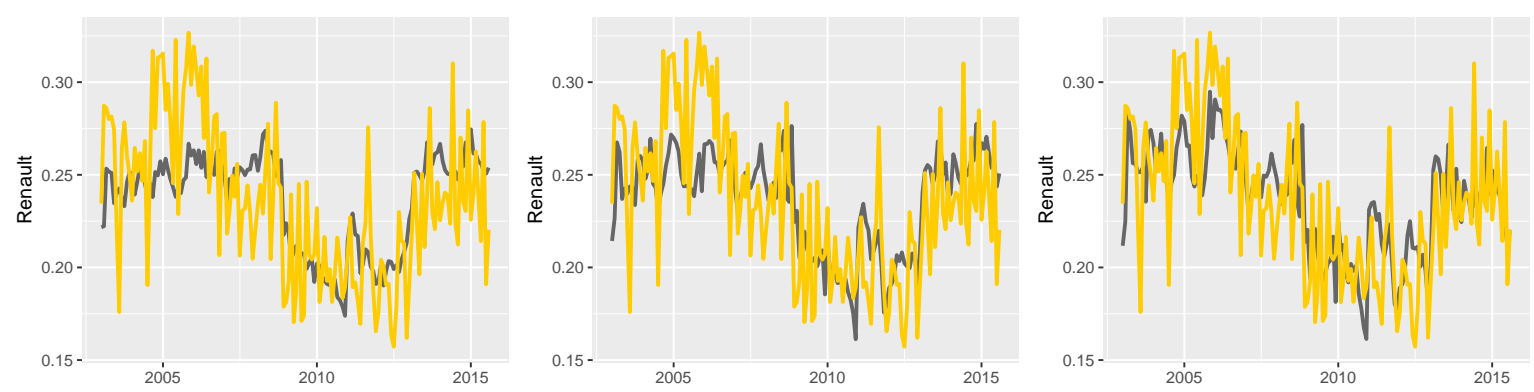

Model A

Model AB

Model B

Serie - Fit - obs
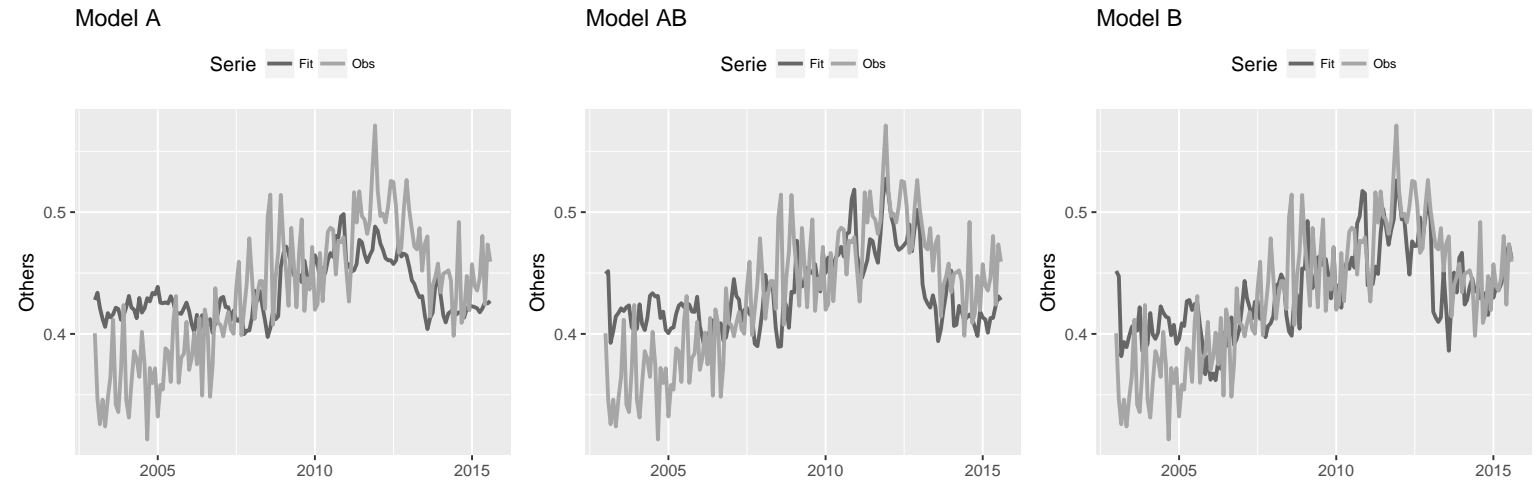

Figure 2: Observed (color) and predicted (grey) brands market-shares

\subsection{Brand-specific impact of media investments (Model B)}

Model Now, let us look at a different specification of the model (dependent and explanatory variables are the same as in Model A) where brand-specific coefficients are assumed and cross- 
$\frac{\text { Table 4: Estimated parameters in the simplex - Model A }}{S_{1}}$

\begin{tabular}{lcccc} 
& (Citroen) & (Peugeot) & (Renault) & (Others) \\
\hline (Intercept) & 0.1300 & 0.2114 & 0.2502 & 0.4084 \\
$M_{t-1}$ & \multicolumn{4}{c}{0.0267} \\
$M_{t-2}$ & \multicolumn{4}{c}{0.0241} \\
$M_{t-3}$ & \multicolumn{4}{c}{0264} \\
$P_{t}$ & \multicolumn{5}{c}{1.2217} \\
$S I$ & 0.2610 & 0.2523 & 0.2086 & 0.2780 \\
\hline
\end{tabular}

effects are directly modeled. It corresponds to the following model:

$$
\begin{gathered}
\mathbf{S}_{t}=\mathbf{a} \bigoplus_{\tau=1}^{3} \mathbf{B}_{\tau} \boxminus \mathbf{M}_{t-\tau} \oplus \mathbf{B}_{\mathbf{P}} \odot \mathbf{P}_{t} \oplus S I_{t} \odot \mathbf{c} \oplus \boldsymbol{\varepsilon}_{t} \\
\Leftrightarrow S_{j t}=\frac{a_{j} \prod_{\tau=1}^{3} \prod_{l=1}^{4} M_{t-\tau, l}^{b_{\tau, j l}} \prod_{l=1}^{4} P_{t, l}^{b_{P, j l}} c_{j}^{S I} \varepsilon_{j t}}{\sum_{m=1}^{4} a_{m} \prod_{\tau=1}^{3} \prod_{l=1}^{4} M_{t-\tau, l}^{b_{\tau, m l}} \prod_{l=1}^{4} P_{t, l}^{b_{P, m l}} c_{m}^{S I} \varepsilon_{m t}}
\end{gathered}
$$

where $\mathbf{B}_{\tau}, \mathbf{B}_{\mathbf{P}} \in \mathbb{R}^{D \times D}$ are the matrices of parameters associated to compositional explanatory variables.

The corresponding ILR transformed model is:

$$
\begin{aligned}
\mathbf{S}_{t}^{*} & =\mathbf{a}^{*}+\sum_{\tau=1}^{3} \mathbf{B}_{\tau}^{*} \mathbf{M}_{t-\tau}^{*}+\mathbf{B}_{\mathbf{P}}^{*} \mathbf{P}_{t}^{*}+\mathbf{c}^{*} S I_{t}+\varepsilon_{t}^{*} \\
\Leftrightarrow S_{j t}^{*} & =a_{j}^{*}+\sum_{\tau=1}^{3} \sum_{l=1}^{3} b_{\tau, j l}^{*} M_{l, t-\tau}^{*}+\sum_{l=1}^{3} b_{P, j l}^{*} P_{l t}^{*}+c_{j}^{*} S I_{t}+\varepsilon_{j t}^{*} \quad \text { for } j=1,2,3
\end{aligned}
$$

where $\varepsilon^{*}$ is supposed to be a Gaussian distributed error term. The same balance matrix $V_{I L R, 4}$ is used.

Results All variables of the model are significant at $0.1 \%$ according to the ANOVA, except the price which is significant at 1\%. According to Figure 2, Model B seems to fit better than Model A (see Section 4.3 for associated quality measures). The estimated parameters of the models are given in Table 5 and Table 6.

\subsection{Interpretation of models $A$ and $B$}

Marginal effect of media investments We calculate the marginal effects of media investments at time $t-1$ on market-shares at time $t$. The average marginal effects are reported in Table 7. They are quite consistent between Model A and Model B, with positive direct marginal effects and negative cross marginal effects. However, these measures are not really adapted to summarize an impact as they fluctuate a lot across time, as we can see in Figure 3 (marginal effects can be larger than $6 \mathrm{e}-08$ but we voluntarily cropped the graph). The marginal effects of Citroen media investments are especially very high when these investments are very low, for example between 2007 and 2009.

Elasticity of the share $S_{j}$ relative to $X_{l}$ For Model A, cross elasticities are necessarily negative and direct elasticities are necessarily positive if the parameter $b$ is positive. Moreover, cross-elasticities of market-shares $S_{j}$ with respect to a particular media budget $M_{l, t-1}$ are equal for any brand $j \neq l$. This is a lack of flexibility of Model A compared to Model B: it does not allow positive interaction between brands, and it considers that if a brand increases 
Table 5: Estimated parameters on ILR coordinates - Model B

\begin{tabular}{|c|c|c|c|}
\hline & $\begin{array}{c}S_{1}^{*} \\
\text { (Peu. vs Cit.) }\end{array}$ & $\begin{array}{c}S_{2}^{*} \\
\text { (Reu. vs Cit.,Peu.) }\end{array}$ & $\begin{array}{c}S_{3}^{*} \\
\text { (Oth. vs Cit.,Peu.,Reu.) }\end{array}$ \\
\hline (Intercept) & $0.3686^{* * *}$ & $0.3637^{* * *}$ & $0.6940^{* * *}$ \\
\hline$M_{t-1,1}^{*}$ & 0.0193. & -0.0052 & 0.0081 \\
\hline$M_{t-1,2}^{*}$ & 0.0162 & $0.0319^{*}$ & -0.0245 \\
\hline$M_{t-1,3}^{*}$ & -0.0069 & 0.0009 & 0.0279 \\
\hline$M_{t-2,1}^{*}$ & 0.0208. & -0.0093 & 0.0205. \\
\hline$M_{t-2,2}^{*}$ & 0.0151 & $0.0361^{* *}$ & -0.0259 . \\
\hline$M_{t-2,3}^{*}$ & -0.0197 & -0.0338 & 0.0278 \\
\hline$M_{t-3,1}^{*}$ & $0.0289^{* *}$ & -0.0115 & $0.0278^{*}$ \\
\hline$M_{t-3,2}^{*}$ & 0.0104 & $0.0206^{*}$ & -0.0274 \\
\hline$M_{t-3,3}^{*}$ & -0.0114 & 0.0064 & 0.0323 . \\
\hline$P_{1}^{*}$ & 0.8854 . & -0.5981 & $1.9138^{* * *}$ \\
\hline$P_{2}^{*}$ & 0.0151 & 0.2615 & 0.6509 \\
\hline$P_{3}^{*}$ & -0.6442 & -0.3729 & $2.4717^{* * *}$ \\
\hline$S I^{*}$ & -0.0394 & $-0.2088^{* * *}$ & $0.2070^{* * *}$ \\
\hline Adjusted R2 & 0.3353 & 0.3255 & 0.3269 \\
\hline Nb param. & 42 & & \\
\hline
\end{tabular}

Table 6: Estimated parameters of $M_{t-1}$ in the simplex - Model B

\begin{tabular}{ccccc}
\hline & $\begin{array}{c}S_{1} \\
(\text { Citroen })\end{array}$ & $\begin{array}{c}S_{2} \\
\text { (Peugeot) }\end{array}$ & $\begin{array}{c}S_{3} \\
\text { (Renault) }\end{array}$ & $\begin{array}{c}S_{4} \\
\text { (Others) }\end{array}$ \\
\hline$M_{t-1,1}$ & 0.0179 & -0.0079 & -0.0067 & -0.0032 \\
$M_{t-1,2}$ & -0.0016 & 0.0111 & -0.0161 & 0.0066 \\
$M_{t-1,3}$ & -0.0132 & 0.0084 & 0.0292 & -0.0243 \\
$M_{t-1,4}$ & -0.0030 & -0.0115 & -0.0064 & 0.0209 \\
\hline
\end{tabular}

Table 7: Average marginal effects of media investments $\check{M}_{t-1}$ on market-shares

\begin{tabular}{l|cccc|cccc}
\hline me $\left(S_{j t}, \check{M}_{l, t-1}\right)$ & \multicolumn{6}{|c}{ Model A } & \multicolumn{4}{c}{ Model B } \\
& $\check{M}_{C, t-1}$ & $\check{M}_{P, t-1}$ & $\check{M}_{R, t-1}$ & $\check{M}_{Z, t-1}$ & $\check{M}_{C, t-1}$ & $\check{M}_{P, t-1}$ & $\check{M}_{R, t-1}$ & $\check{M}_{Z, t-1}$ \\
\hline$S_{\text {Citroen }, t}$ & $\mathbf{1 . 9 3 e - 0 5}$ & $-1.65 \mathrm{e}-09$ & $-2.13 \mathrm{e}-09$ & $-3.01 \mathrm{e}-10$ & $\mathbf{1 . 6 8 e - 0 5}$ & $-7.20 \mathrm{e}-10$ & $-2.82 \mathrm{e}-09$ & $-2.00 \mathrm{e}-10$ \\
$S_{\text {Peugeot }, t}$ & $-4.58 \mathrm{e}-06$ & $\mathbf{1 . 1 4 e - 0 8}$ & $-3.09 \mathrm{e}-09$ & $-5.30 \mathrm{e}-10$ & $-7.67 \mathrm{e}-06$ & $\mathbf{5 . 5 1 e - 0 9}$ & $7.72 \mathrm{e}-09$ & $-7.52 \mathrm{e}-10$ \\
$S_{\text {Renault }, t}$ & $-4.88 \mathrm{e}-06$ & $-3.64 \mathrm{e}-09$ & $\mathbf{1 . 3 5 e - 0 8}$ & $-5.96 \mathrm{e}-10$ & $-6.43 \mathrm{e}-06$ & $-1.14 \mathrm{e}-08$ & $\mathbf{2 . 2 3 e - 0 8}$ & $-5.71 \mathrm{e}-10$ \\
$S_{\text {Others }, t}$ & $-9.89 \mathrm{e}-06$ & $-6.10 \mathrm{e}-09$ & $-8.24 \mathrm{e}-09$ & $\mathbf{1 . 4 3 e - 0 9}$ & $-2.66 \mathrm{e}-06$ & $6.60 \mathrm{e}-09$ & $-2.72 \mathrm{e}-08$ & $\mathbf{1 . 5 2 e}-\mathbf{0 9}$ \\
\hline
\end{tabular}

C: Citroen; P: Peugeot; R: Renault; Z: Others.

Figures in bold: direct elasticities.

Model A

Brand Citroen - Peugeot - Renault $2 z z$

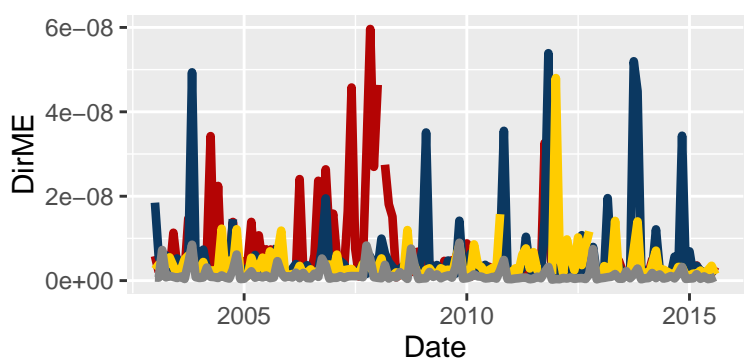

Model B

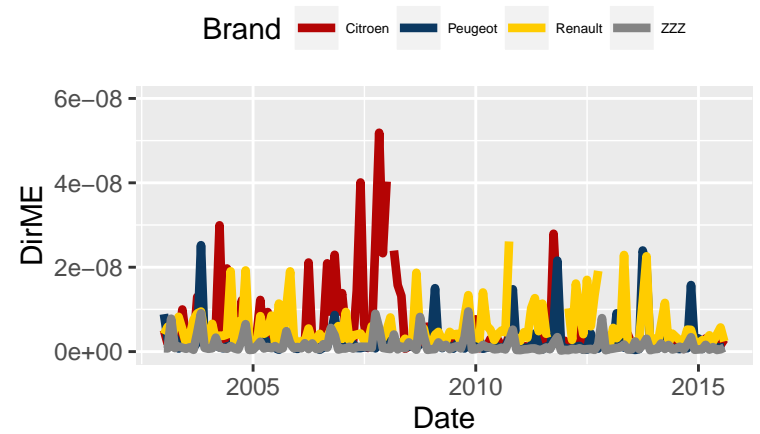

Figure 3: Direct marginal effects of $M_{j, t-1}$ on $S_{j t}$ across time

its media investments of $1 \%$ it will affect in the same way all competitors market-shares $S_{j}$ (they will all decrease by $b \%$ ). 
Let us consider a situation where the market shares of Citroen, Peugeot, Renault and Others in the B segment are respectively 10\%, 25\%, 25\% and 40\%. According to Table 8, if Renault increases its media investments $M_{t-1}$ about $1 \%$, the average elasticity of Model A on the studied period suggests that its market-share should increase by $0.0204 \%$ to reach $25.005 \%$ and that competitors market-shares should decrease by $0.0204 \%$ to reach respectively $9.998 \%$, $24.995 \%$ and $39.992 \%{ }^{10}$.

In Model B, when brand-specific effects and cross-effects are taken into account, the direct elasticity of Renault market-share in the B segment relative to its corresponding media investments (0.0327) is much higher than for other brands, see for example Peugeot which has the lowest (0.0099). Note that positive cross-effects (synergies) are possible in Model B: for example when Renault invests more in media, it tends to help its own market-share a lot, but also to raise a little bit the share of Peugeot, and to have a negative impact on Citroen and Others. Then, after closure and depending on the considered values of $S_{j}$, an increase in Renault media investments in the B segment can increase or decrease the Peugeot market-share.

Taking the same example as previously, according to Model B, if Renault increases its media investments $M_{t-1}$ of about $1 \%$, the average elasticity on the studied period suggests that its market-share should increase by $0.0327 \%$ to reach $25.008 \%$ and that competitors marketshares should respectively decrease by $0.0097 \%$, increase by $0.0119 \%$ and decrease by $0.0208 \%$ to reach respectively $9.999 \%, 25.003 \%$ and 39.992 .

As shown in Figure 4, the estimated direct elasticities are quite stable across time. However, as elasticities in Model A are computed using the same parameter $b$ for all brands, they are closer to each other than in Model B where they are computed using different parameters $b_{j l}$. The direct elasticity of Renault is larger than those of other brands during the whole studied period.
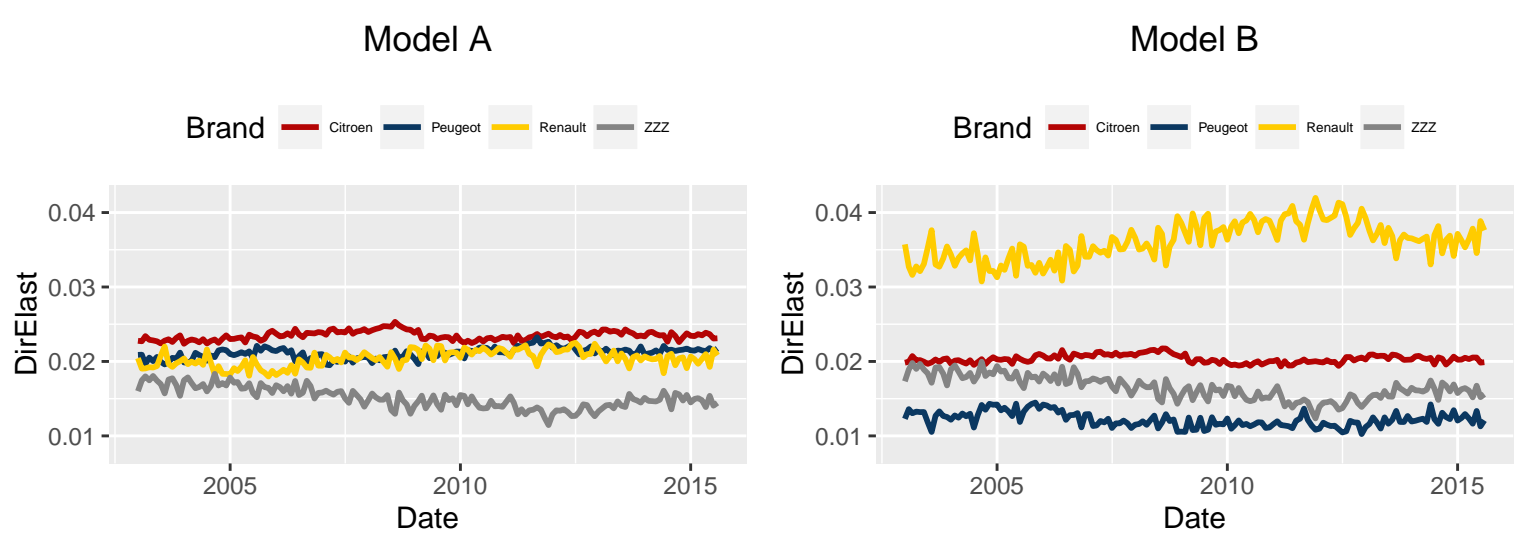

Figure 4: Direct elasticity of $S_{j t}$ relative to $M_{j, t-1}$ across time

\section{Elasticity of the ratio $\frac{S_{j}}{S_{j^{\prime}}}$ relative to $\check{X}_{l} \quad$ (see Table 9)}

In Model A, the elasticity of a ratio $S_{j} / S_{j^{\prime}}$ relative to $\check{X}_{j}$ is equal to 0.0267 , whereas in Model $\mathrm{B}$ it can be smaller or larger according to the considered brands: the largest elasticity is for $S_{R} / S_{Z}$ relative to $\check{X}_{R}$ which is equal to 0.0535 . In general, ratios between the market-share of Renault and another brand are quite positively sensitive to media investments of Renault. For example, if the ratio $S_{R} / S_{Z}$ is equal to $25 / 40=0.6250$ and Renault increases by $1 \%$ its

\footnotetext{
${ }^{10} \mathrm{NB}$ : here we take an example for an arbitrary share of $25 \%$ using the average elasticity. However, the only way to ensure that the sum of the modified shares $\sum_{m=1}^{D} S_{m t}^{\prime}$ is equal to 1 is to use the corresponding elasticities calculated at the same time $t$, not the average elasticities.
} 
Table 8: Average elasticity of market-shares relative to media investments $\check{M}_{t-1}$

\begin{tabular}{l|cccc|cccc}
\hline$e\left(S_{j t}, \check{M}_{l, t-1}\right)$ & \multicolumn{6}{|c}{ Model A } & \multicolumn{5}{c}{ Model B } \\
& $\check{M}_{C, t-1}$ & $\check{M}_{P, t-1}$ & $\check{M}_{R, t-1}$ & $\check{M}_{Z, t-1}$ & $\check{M}_{C, t-1}$ & $\check{M}_{P, t-1}$ & $\check{M}_{R, t-1}$ & $\check{M}_{Z, t-1}$ \\
\hline$S_{\text {Citroen }, t}$ & $\mathbf{0 . 0 2 3 5}$ & -0.0056 & -0.0063 & -0.0116 & $\mathbf{0 . 0 2 0 4}$ & -0.0028 & -0.0097 & -0.0078 \\
$S_{\text {Peugeot }, t}$ & -0.0032 & $\mathbf{0 . 0 2 1 1}$ & -0.0063 & -0.0116 & -0.0054 & $\mathbf{0 . 0 0 9 9}$ & 0.0119 & -0.0163 \\
$S_{\text {Renault }, t}$ & -0.0032 & -0.0056 & $\mathbf{0 . 0 2 0 4}$ & -0.0116 & -0.0043 & -0.0173 & $\mathbf{0 . 0 3 2 7}$ & -0.0111 \\
$S_{\text {Others }, t}$ & -0.0032 & -0.0056 & -0.0063 & $\mathbf{0 . 0 1 5 1}$ & -0.0008 & 0.0054 & -0.0208 & $\mathbf{0 . 0 1 6 1}$ \\
\hline
\end{tabular}

C: Citroen; P: Peugeot; R: Renault; Z: Others.

Figures in bold: direct elasticities.

media investments, then the ratio will increase to 0.6253. Let us remind that this measure does not depend on the considered period. This evolution is consistent with the fact that the market-share of Renault is very positively elastic and the market-share of "Others" is very negatively elastic to Renault media investments, as seen in Table 8.

Table 9: Elasticity of ratios of market-shares $\frac{S_{j t}}{S_{j^{\prime} t}}$ relative to media investments $\check{M}_{l, t-1}$

\begin{tabular}{|c|c|c|c|c|c|c|c|c|c|}
\hline \multirow{2}{*}{\multicolumn{2}{|c|}{ Model A }} & \multicolumn{8}{|c|}{ Model B } \\
\hline & & & $\check{M}_{C, t-1}$ & & $\check{M}_{P, t-1}$ & & $\check{M}_{R, t-1}$ & & $\check{M}_{Z, t-1}$ \\
\hline$e\left(\frac{S_{j t}}{S_{j^{\prime} t}}, \check{M}_{j, t-1}\right)$ & 0.0267 & $S_{C / P}$ & 0.0258 & $S_{P / C}$ & 0.0127 & $S_{R / C}$ & 0.0424 & $S_{Z / C}$ & 0.0239 \\
\hline$e\left(\frac{S_{j t}}{S_{j^{\prime} t}}, \check{M}_{j^{\prime}, t-1}\right)$ & -0.0267 & $S_{C / R}$ & 0.0246 & $S_{P / R}$ & 0.0272 & $S_{R / P}$ & 0.0208 & $S_{Z / P}$ & 0.0325 \\
\hline$e\left(\frac{S_{j^{\prime}}}{S_{j^{\prime} t}}, \check{M}_{l, t-1}\right)^{*}$ & 0 & $S_{C / Z}$ & 0.0211 & $S_{P / Z}$ & 0.0044 & $S_{R / Z}$ & 0.0535 & $S_{Z / R}$ & 0.0273 \\
\hline
\end{tabular}

${ }^{*}$ where $l \neq j, j^{\prime}$ and $S_{C / Z}$ means $S_{\text {Citroen }, t} / S_{\text {Others }, t}$ for example.

Odds ratio of $\frac{S_{j}}{S_{j^{\prime}}}$ to a change of $\check{X}_{l} \quad$ (see Table 10)

As expected, this measure is consistent with the previous one. In Model A, the odds ratio of any couple of brand market-shares $S_{j} / S_{j^{\prime}}$ to a change of $10 \%$ of $\check{M}_{j, t-1}$ is equal to 1.0025 , whereas it can reach 1.0054 in Model B for the ratio $S_{R} / S_{Z}$ for a change of $10 \%$ in $\check{M}_{R, t-1}$. It means that if the ratio of market-shares of Renault over Others is equal to $25 / 40=0.6250$ and Renault decides to increase its media budget by $10 \%$, then this ratio will increase to 0.6266 according to Model A and to 0.6284 according to Model B.

Table 10: Odds ratios of market-shares for an increase of $10 \%$ in media investments $\check{M}_{l, t-1}$

\begin{tabular}{|c|c|c|c|c|c|c|c|c|c|}
\hline \multicolumn{2}{|l|}{ Model A } & \multicolumn{8}{|c|}{ Model B } \\
\hline For $\Delta=10 \%$ & $\check{M}_{t-1}$ & & $\check{M}_{C, t-1}$ & & $\check{M}_{P, t-1}$ & & $\check{M}_{R, t-1}$ & & $\check{M}_{Z, t-1}$ \\
\hline $\operatorname{OR}\left(\frac{S_{j t}}{S_{j^{\prime} t}}, \check{M}_{j, t-1}, \Delta\right)$ & 1.0025 & $S_{C / P}$ & 1.0025 & $S_{P / C}$ & 1.0012 & $S_{R / C}$ & 1.0045 & $S_{Z / C}$ & 1.0022 \\
\hline $\mathrm{OR}\left(\frac{S_{j_{t}}}{S_{j^{\prime}}}, \check{M}_{j^{\prime}, t-1}, \Delta\right)$ & 0.9975 & $S_{C / R}$ & 1.0024 & $S_{P / R}$ & 1.0030 & $S_{R / P}$ & 1.0026 & $S_{Z / P}$ & 1.0031 \\
\hline $\operatorname{OR}\left(\frac{S_{j t}}{S_{j^{\prime} t}}, \check{M}_{l, t-1}, \Delta\right)^{*}$ & 0 & $S_{C / Z}$ & 1.0020 & $S_{P / Z}$ & 1.0007 & $S_{R / Z}$ & 1.0054 & $S_{Z / R}$ & 1.0028 \\
\hline
\end{tabular}

${ }^{*}$ where $l \neq j, j^{\prime}$ and $S_{C / Z}$ means $S_{\text {Citroen }, t} / S_{\text {Others }, t}$ for example.

Elasticity of $\frac{S_{j}}{g\left(S_{-j}\right)}$ relative to $\check{X}_{l} \quad$ (see Table 11)

As in Model A, no matter which transformation is used, the parameter $b_{1}$ will be the same, then we obtain that $e\left(\frac{S_{j t}}{g\left(S_{-j t}\right)}, \frac{M_{j, t-1}}{g\left(M_{-j, t-1}\right)}\right)=e\left(\frac{S_{j t}}{S_{j^{\prime} t}}, M_{j, t-1}\right)=e\left(\frac{S_{j t}}{S_{j^{\prime} t}}, \frac{M_{j, t-1}}{M_{j^{\prime}, t-1}}\right)$. Moreover, these elasticities are consistent with previous impact measures, and the largest one concerns the ratio $\frac{S_{R}}{g\left(S_{-R}\right)}$ relatively to the ratio $\frac{M_{R}}{g\left(M_{-R}\right)}$, which is equal to $0.0389 \%$. For example, let us consider a situation where the market-shares are the following: $\left(S_{C}, S_{P}, S_{R}, S_{Z}\right)^{\prime}=$ $(13,22,25,40)^{\prime}$, inducing that $\frac{S_{R}}{g\left(S_{-R}\right)}=1.1095$. Then, if Renault increases its media investments by $1 \%$ of the geometric average of other brands media investments, we can expect its 
market-share to move from $110.95 \%$ to $110.99 \%$ of the geometric average market-share of others.

\begin{tabular}{l} 
Table 11: Elasticity of ratios $\frac{S_{j t}}{g\left(S_{-j t)}\right.}$ relative to $\check{M}_{l, t-1}$ \\
\hline Model A \\
\cline { 3 - 6 }
\end{tabular}

\subsection{Complexity and goodness-of-fit}

We have seen that Model A and Model B can be used for the same type of application. Model $\mathrm{B}$ is more complex than Model A because it allows to have component-specific parameters for each explanatory variables along with cross-effects parameters. We have also fitted an intermediate model without component-specific and cross-effects parameters for the price (Model AB). The number of parameters to fit of Model B can be a serious limitation when the number of components $D$ and the number of explanatory compositions $K$ increase. For example, in our application Model A involves 10 parameters whereas Model AB and Model B involve respectively 34 and 42 parameters.

However, Model B is also more flexible than Model A in the sense that it allows to have positive synergies (positive interactions) between some shares, whereas cross elasticities of Model A are necessarily negative ${ }^{11}$. For example, we see in Table 8 that when the media investments of Citroen increase, it tends to benefit also to "Others", and when the media investments of Renault increase, it tends to benefit to Peugeot.

Is the complexity of Model B useful to explain brands market-shares of the B segment? According to the Fisher tests of Model A against Model B, and Model AB against Model $\mathrm{B}$, for which the estimated statistics are respectively 2.22 and 3.72 to be compared to the $99 \%$ quantiles, respectively 0.51 and 0.56 , we conclude that Model B is significantly more adapted than Model A and Model AB. This means that the brand specific and cross effect parameters for media investments and prices are necessary to reflect the complexity of the competitive interaction in the automobile market. We also compare cross-validated ${ }^{12}$ quality measures: adjusted $R^{2}$ calculated on the transformed model with coordinates used for the estimation ${ }^{13}, R^{2}$ based on the total variance (as defined in compositional data analysis, see van den Boogaart and Tolosana-Delgado (2013)) and RMSE (see Table 12). All measures agree on the fact that Model B is better than Model A and Model AB for our application.

Table 12: Cross-validated quality measures

\begin{tabular}{rrrr}
\hline & Model A & Model AB & Model B \\
\hline Adj. $R^{2}$ & 0.9250 & 0.9274 & $\mathbf{0 . 9 3 1 0}$ \\
$R_{T}^{2}$ & 0.3183 & 0.4002 & $\mathbf{0 . 4 5 1 3}$ \\
RMSE & 0.0326 & 0.0913 & $\mathbf{0 . 0 3 2 2}$ \\
\hline
\end{tabular}

\footnotetext{
${ }^{11}$ As long as the direct elasticity is positive (the cross elasticity is of opposite sign of the direct elasticity by construction).

${ }^{12}$ The out-of-sample computation process is the same than in Morais et al. (2017).

${ }^{13}$ For Model $\mathrm{B}$, the adjusted $R^{2}$ is computed on the transformed model which uses dummy variables for estimations, as in Model A and Model AB.
} 


\section{Conclusion}

The focus of this paper is to present of compositional models for the case when the dependent variable and some of the explanatory variables are compositions, and to interpret them. The difference between Model A (coming from marketing) and Model B (coming from CODA) is due to the model specification: in Model A, a single global coefficient is associated to an explanatory composition, whereas in Model B we assume that each component of the explanatory composition has a specific impact on each component of the dependent variable. In Model B, cross-effects between components are explicitly specified and can be positive, whereas in Model A(coming from marketing) they are implicit and negative by construction. Consequently, Model B is more flexible but also much more complex than Model A, and the number of parameters to fit can be a serious limitation to use it. We prove in this paper that Model A is a constrained version of Model B, and that an intermediate Model AB can also be considered. An adapted Fisher test can be used for model selection.

This paper also presents a set of possible measures, mutually consistent, to interpret parameters of these two models: marginal effects, elasticities and odds ratios. Elasticities are totally adapted to enhance the interpretability of these models. However, this measure is observation dependent and we have to make sure that it is stable across observations to use it. Marginal effects are not well adapted to interpret this kind of models because they depend a lot on the considered observation. The other types of measures presented have the advantage to be observation independent, but they are more difficult to interpret in practical cases because they involve ratios.

The two models and an intermediate specification are applied to the B segment of the French automobile market, for the purpose of measuring the impact of brand media investments on brands market-shares. Model B fits our data better than Model A and Model AB according to cross-validated quality measures and to Fisher tests. In Model B, Renault is the brand which has the largest direct elasticity to media investments. This model also shows interesting non-symmetric synergies between brands.

In a further work, as compositions are observed across time, the potential autocorrelation of error terms has to be considered. Moreover, from a marketing point of view, it would be interesting to measure the impact of each channel (TV, radio, press, outdoor, internet, cinema) separately.

Acknowledgment: We thank BVA and the Marketing Direction of Renault for sharing valuable data with us, and for their support during the model specification and interpretation. We are very grateful to Josep Antoni Martin-Fernandez, Vera Pawlowsky-Glahn and Juan Jose Egozcue for their feedback and their good advice.

Funding: This work was supported by the market research agency BVA and the French national research agency ANRT.

\section{References}

Aitchison J (1986). The Statistical Analysis of Compositional Data. Monographs on Statistics and Applied Probability. Chapman and Hall. ISBN 9780412280603. URL https://books . google.fr/books?id=RHKmAAAAIAAJ.

Barcelo-Vidal C, Martín-Fernández JA (2016). "The Mathematics of Compositional Analysis." Austrian Journal of Statistics, 45(4), 57-71.

Chen J, Zhang X, Li S (2017). "Multiple Linear Regression with Compositional Response and Covariates." Journal of Applied Statistics, 44(12), 2270-2285. 
Cooper L, Nakanishi M (1988). Market-Share Analysis: Evaluating Competitive Marketing Effectiveness. International Series in Quantitative Marketing. Springer. ISBN 9780898382785. URL https://books.google.fr/books?id=d91YDCfF0zMC.

Egozcue JJ, Pawlowsky-Glahn V, Mateu-Figueras G, Barcelo-Vidal C (2003). "Isometric Logratio Transformations for Compositional Data Analysis." Mathematical Geology, 35(3), 279-300.

Fišerová E, Hron K (2011). "On the Interpretation of Orthonormal Coordinates for Compositional Data." Mathematical Geosciences, 43(4), 455.

Hron K, Filzmoser P, Thompson K (2012). "Linear Regression with Compositional Explanatory Variables." Journal of Applied Statistics, 39(5), 1115-1128.

Mateu-Figueras G, Pawlowsky-Glahn V (2008). "A Critical Approach to Probability Laws in Geochemistry." In Progress in Geomathematics, pp. 39-52. Springer.

Morais J, Thomas-Agnan C, Simioni M (2016). "A Tour of Regression Models for Explaining Shares." TSE Working Paper, (16-742). URL https://www.tse-fr.eu/fr/publications/ tour-regression-models-explaining-shares.

Morais J, Thomas-Agnan C, Simioni M (2017). "Using Compositional and Dirichlet Models for Market Share Regression." Journal of Applied Statistics. doi:10.1080/02664763.2017. 1389864.

Muller I, Hron K, Fiserova E, Smahaj J, Cakirpaloglu P, Vancakova J (2015). "Time Budget Analysis Using Logratio Methods."

Pawlowsky-Glahn V, Buccianti A (2011). Compositional Data Analysis: Theory and Applications. John Wiley \& Sons.

Pawlowsky-Glahn V, Egozcue JJ, Tolosana-Delgado R (2015). Modeling and Analysis of Compositional Data. John Wiley \& Sons.

van den Boogaart KG, Tolosana-Delgado R (2013). Analysing Compositional Data with R. Springer.

Wang H, Shangguan L, Wu J, Guan R (2013). "Multiple Linear Regression Modeling for Compositional Data." Neurocomputing, 122, 490-500. 


\section{A. Appendix}

\section{A.1. Model A is a particular case of Model B}

Model A is a particular case of Model B if and only if one can show that $b \odot \mathbf{X}$ can be equal to $\mathbf{B} \sqcup \mathbf{X}$ :

$$
\begin{aligned}
b \odot \mathbf{X}=\mathbf{B} \odot \mathbf{X} & \Leftrightarrow i \operatorname{lr}(b \odot \mathbf{X})=i \operatorname{lr}(\mathbf{B} \odot \mathbf{X}) \\
& \Leftrightarrow b i l r(\mathbf{X})=\mathbf{B}^{*} i \operatorname{lr}(\mathbf{X}) \\
& \Leftrightarrow b \mathbf{I d}_{\mathbf{D}-\mathbf{1}}=\mathbf{B}^{*}
\end{aligned}
$$

where $\mathbf{I d}_{\mathbf{D}-\mathbf{1}}$ is the identity matrix of dimension $D-1$ when $\mathbf{B}$ is of dimension $D \times D$ (let us remind that Model A can be used only when dependant and explanatory compositions have the same dimension $D$ ). Indeed, Model $\mathbf{A}$ is such that $\mathbf{B}^{*}$ is diagonal with diagonal terms equal to $b$. This relationship holds for any ILR transformation.

\section{A.2. Marginal effect and elasticity calculus on ILR}

We are going to demonstrate how to compute marginal effects of the volume $\check{X}_{l t}$ on the dependent shares $S_{j t}$, and elasticities of $S_{j t}$ relative to $\check{X}_{l t}$, using the transformed and the non-transformed models. The demonstration is made for Model $\mathrm{B}$, with $D=3$ components and a pivot ILR transformation using the matrix $\mathbf{V}_{I L R, 3}$ as in Appendix A.1, but it holds for any ILR transformation. Let us remind that $\mathbf{X}^{*}=i \operatorname{lr}(\mathbf{X})=\mathbf{V}^{\prime} \log (\mathbf{X})$, and $\mathbf{X}=i l r^{-1}\left(\mathbf{X}^{*}\right)=$ $\mathcal{C}\left(\exp \left(\mathbf{V X}^{*}\right)\right)$.

We define the following transformations:

$$
\begin{aligned}
T & :\left(\check{X}_{1}, \check{X}_{2}, \check{X}_{3}\right)^{\prime} \rightarrow\left(\check{X}_{1}^{*}, \check{X}_{2}^{*}\right)^{\prime} \\
F & :\left(\check{X}_{1}^{*}, \check{X}_{2}^{*}\right)^{\prime} \rightarrow\left(\mathbb{E} S_{1}^{*}, \mathbb{E} S_{2}^{*}\right)^{\prime}=\left(a_{1}^{*}+b_{11}^{*} \check{X}_{1}^{*}+b_{12}^{*} \check{X}_{2}^{*}, a_{2}^{*}+b_{21}^{*} \check{X}_{1}^{*}+b_{22}^{*} \check{X}_{2}^{*}\right)^{\prime} \\
T^{-1} & :\left(\mathbb{E} S_{1}^{*}, \mathbb{E} S_{2}^{*}\right)^{\prime} \rightarrow\left(\mathbb{E}^{\oplus} S_{1}, \mathbb{E}^{\oplus} S_{2}, \mathbb{E}^{\oplus} S_{3}\right)^{\prime}
\end{aligned}
$$

We are going to use the following property of Jacobian matrices: $J=J_{T^{-1}} J_{F} J_{T}$, implying that:

$$
M E\left(\mathbb{E}^{\oplus} \mathbf{S}_{t}, \check{\mathbf{X}}_{t}\right)=\left[\frac{\partial \mathbb{E}^{\oplus} S_{i t}}{\partial \check{X}_{j t}}\right]_{D, D}=\left[\frac{\partial \mathbb{E}^{\oplus} S_{i t}}{\partial \mathbb{E} S_{j t}^{*}}\right]_{D, D-1}\left[\frac{\partial \mathbb{E} S_{i t}^{*}}{\partial \check{X}_{j t}^{*}}\right]_{D-1, D-1}\left[\frac{\partial \check{X}_{i t}^{*}}{\partial \check{X}_{j t}}\right]_{D-1, D}
$$

and

$E\left(\mathbb{E}^{\oplus} \mathbf{S}_{t}, \check{\mathbf{X}}_{t}\right)=\left[\frac{\partial \log \mathbb{E}^{\oplus} S_{i t}}{\partial \log \check{X}_{j t}}\right]_{D, D}=\left[\frac{\mathbf{1}}{\mathbf{S}_{\mathbf{i t}}}\right] \odot\left[\frac{\partial \mathbb{E}^{\oplus} S_{i t}}{\partial \mathbb{E} S_{j t}^{*}}\right]_{D, D-1}\left[\frac{\partial \mathbb{E} S_{i t}^{*}}{\partial \check{X}_{j t}^{*}}\right]_{D-1, D-1}\left[\frac{\partial \check{X}_{i t}^{*}}{\partial \check{X}_{j t}}\right]_{D-1, D} \odot\left[\mathbf{X}_{\mathbf{j t}}\right]$

where $\odot$ denotes here the Hadamard product (term by term product) ${ }^{14},\left[\frac{\mathbf{1}}{\mathbf{S}_{\mathrm{it}}}\right]$ is a $D \times D-1$ matrix with $1 / S_{i t}$ on the $i^{\text {th }}$ row and $\left[\mathbf{X}_{\mathbf{j t}}\right]$ is a $D-1, D$ matrix with $X_{j t}$ on the $j^{\text {th }}$ column.

The Jacobian of the model in coordinates $J_{F}$

$$
J_{F}=\left[\begin{array}{ll}
\frac{\partial \mathbb{E} S_{1}^{*}}{\partial \dot{X}_{1}^{*}} & \frac{\partial \mathbb{E} S_{1}^{*}}{\partial \dot{X}_{2}^{*}} \\
\frac{\partial \mathbb{E} S_{2}^{*}}{\partial \dot{X}_{1}^{*}} & \frac{\partial \mathbb{E} S_{2}^{*}}{\partial \dot{X}_{2}^{*}}
\end{array}\right]=\left[\begin{array}{ll}
b_{11}^{*} & b_{12}^{*} \\
b_{21}^{*} & b_{22}^{*}
\end{array}\right]=\mathbf{B}^{*}
$$

\footnotetext{
${ }^{14}$ Note that $\odot$ in bold denote the Hadamard product whereas $\odot$ denote the power transformation.
} 
The Jacobian of the transformation $J_{T}$ The ILR transformation is defined by:

$\left(\check{X}_{1}^{*}, \check{X}_{2}^{*}\right)^{\prime}=T\left(\check{X}_{1}, \check{X}_{2}, \check{X}_{3}\right)^{\prime}=\left(\sqrt{\frac{2}{3}} \log \check{X}_{1}-\frac{1}{\sqrt{6}} \log \check{X}_{2}-\frac{1}{\sqrt{6}} \log \check{X}_{3}, \frac{1}{\sqrt{2}} \log \check{X}_{2}-\frac{1}{\sqrt{2}} \log \check{X}_{3}\right)^{\prime}$

Then, $J_{T}=\left[\begin{array}{lll}\frac{\partial \check{X}_{1}^{*}}{\partial \dot{X}_{1}} & \frac{\partial \check{X}_{1}^{*}}{\partial \dot{X}_{2}} & \frac{\partial \check{X}_{1}^{*}}{\partial \dot{X}_{3}} \\ \frac{\partial \dot{X}_{2}^{*}}{\partial \dot{X}_{1}} & \frac{\partial \dot{X}_{2}^{*}}{\partial \dot{X}_{2}} & \frac{\partial \check{X}_{2}^{*}}{\partial \dot{X}_{3}}\end{array}\right]=\mathbf{V}^{\prime} \odot\left[\frac{1}{\mathbf{X}_{\mathbf{j}}}\right]=\left[\begin{array}{ccc}\sqrt{\frac{2}{3}} \frac{1}{X_{1}} & -\frac{1}{\sqrt{6}} \frac{1}{X_{2}} & -\frac{1}{\sqrt{6}} \frac{1}{X_{3}} \\ 0 & \frac{1}{\sqrt{2}} \frac{1}{X_{2}} & -\frac{1}{\sqrt{2}} \frac{1}{X_{3}}\end{array}\right]$

where $\left[\frac{\mathbf{1}}{\mathbf{X}_{\mathbf{j}}}\right]$ is a $D-1, D$ matrix with $1 / X_{j}$ on the $j^{\text {th }}$ column.

The Jacobian of the inverse transformation $J_{T^{-1}}$

$\left(\mathbb{E}^{\oplus} S_{1}, \mathbb{E}^{\oplus} S_{2}, \mathbb{E}^{\oplus} S_{3}\right)^{\prime}=T^{-1}\left(\mathbb{E} S_{1}^{*}, \mathbb{E} S_{2}^{*}\right)^{\prime}=\mathcal{C}\left(\exp \left(\mathbf{V} \mathbb{E} \mathbf{S}^{*}\right)^{\prime}\right)$

$$
\begin{aligned}
& =\mathcal{C}\left(\exp \left(\mathbb{E} S_{1}^{*}\right)^{\sqrt{\frac{2}{3}}} ; \exp \left(\mathbb{E} S_{1}^{*}\right)^{-\frac{1}{\sqrt{6}}} \exp \left(\mathbb{E} S_{2}^{*}\right)^{\frac{1}{\sqrt{2}}} ; \exp \left(\mathbb{E} S_{1}^{*}\right)^{-\frac{1}{\sqrt{6}}} \exp \left(\mathbb{E} S_{2}^{*}\right)^{-\frac{1}{\sqrt{2}}}\right)^{\prime} \\
& =\left(\frac{u_{1}}{D E N} ; \frac{u_{2}}{D E N} ; \frac{u_{3}}{D E N}\right)
\end{aligned}
$$

where

$$
\begin{aligned}
u_{1} & =\exp \left(\mathbb{E} S_{1}^{*}\right)^{\sqrt{\frac{2}{3}}} \\
u_{2} & =\exp \left(\mathbb{E} S_{1}^{*}\right)^{-\frac{1}{\sqrt{6}}} \exp \left(\mathbb{E} S_{2}^{*}\right)^{\frac{1}{\sqrt{2}}} \\
u_{3} & =\exp \left(\mathbb{E} S_{1}^{*}\right)^{-\frac{1}{\sqrt{6}}} \exp \left(\mathbb{E} S_{2}^{*}\right)^{-\frac{1}{\sqrt{2}}} \\
D E N & =u_{1}+u_{2}+u_{3}
\end{aligned}
$$

In order to compute the matrix $J_{T^{-1}}=\left[\begin{array}{cc}\frac{\partial \mathbb{E}^{\oplus} S_{1}}{\partial \mathbb{E} S_{1}^{*}} & \frac{\partial \mathbb{E}^{\oplus} S_{1}}{\partial \mathbb{E} S^{*}} \\ \frac{\partial \mathbb{E}^{\oplus} S_{2}}{\partial \mathbb{E}^{*}} & \frac{\partial \mathbb{E}^{\oplus} S_{2}}{\partial \mathbb{E} S_{2}^{*}} \\ \frac{\partial \mathbb{E}^{\oplus} S_{3}}{\partial \mathbb{E}_{1}^{*}} & \frac{\partial \mathbb{E}^{\oplus} S_{3}}{\partial \mathbb{E} S_{2}^{*}}\end{array}\right]$, we need to compute the derivatives of the numerators of $\mathbb{E}^{\oplus} \mathbf{S}: \mathbf{u}=\left(u_{1}, u_{2}, u_{3}\right)^{\prime}$ with respect to $\mathbb{E} \mathbf{S}^{*}$.

$$
\left(\frac{\partial \mathbf{u}}{\partial \mathbb{E} \mathbf{S}^{*}}\right)=\mathbf{V} \odot \mathbf{u}=\left[\begin{array}{cc}
\frac{\partial u_{1}}{\partial \mathbb{E} S_{1}^{*}}=\sqrt{\frac{2}{3}} u_{1} & \frac{\partial u_{1}}{\partial \mathbb{E} S_{2}^{*}}=0 \\
\frac{\partial u_{2}}{\partial \mathbb{E} S_{1}^{*}}=-\frac{1}{\sqrt{6}} u_{2} & \frac{\partial u_{2}}{\partial \mathbb{E} S_{2}^{*}}=\frac{1}{\sqrt{2}} u_{2} \\
\frac{\partial u_{3}}{\partial \mathbb{E} S_{1}^{*}}=-\frac{1}{\sqrt{6}} u_{3} & \frac{\partial u_{3}}{\partial \mathbb{E} S_{2}^{*}}=-\frac{1}{\sqrt{2}} u_{3}
\end{array}\right]
$$

Now we can compute the elements of $J_{T^{-1}}$. For example, the first element of this matrix is:

$$
\frac{\partial \mathbb{E}^{\oplus} S_{1}}{\partial \mathbb{E} S_{1}^{*}}=\frac{D E N \sqrt{\frac{2}{3}} u_{1}-u_{1}\left[\sqrt{\frac{2}{3}} u_{1}-\frac{1}{\sqrt{6}} u_{2}-\frac{1}{\sqrt{6}} u_{3}\right]}{D E N^{2}}=\frac{\frac{3}{\sqrt{6}} u_{1}\left(u_{2}+u_{3}\right)}{D E N^{2}}=\frac{3}{\sqrt{6}} \mathbb{E}^{\oplus} S_{1}\left(1-\mathbb{E}^{\oplus} S_{1}\right)
$$

using the fact that $u_{1} / D E N=\mathbb{E}^{\oplus} S_{1}$ and $u_{2}+u_{3}=D E N-u_{1}$.

Similar computations give the results for the whole matrix:

$$
\begin{aligned}
J_{T^{-1}} & =\left[\begin{array}{ll}
\frac{\partial \mathbb{E}^{\oplus} S_{1}}{\partial \mathbb{E} S^{*}} & \frac{\partial \mathbb{E}^{\oplus} S_{1}}{\partial \mathbb{E} S^{*}} \\
\frac{\partial \mathbb{E}^{\oplus} S_{2}}{\partial \mathbb{E} S^{*}} & \frac{\partial \mathbb{E}^{\oplus} S_{2}}{\partial \mathbb{E} S^{*}} \\
\frac{\partial \mathbb{E}^{\oplus}}{\partial \mathbb{E} S_{1}^{*}} & \frac{\partial \mathbb{E}^{\oplus}}{\partial \mathbb{E} S_{2}^{*}}
\end{array}\right]=\left[\begin{array}{cc}
\frac{3}{\sqrt{6}} \mathbb{E}^{\oplus} S_{1}\left(1-\mathbb{E}^{\oplus} S_{1}\right) & \frac{1}{\sqrt{2}} \mathbb{E}^{\oplus} S_{1}\left(\mathbb{E}^{\oplus} S_{3}-\mathbb{E}^{\oplus} S_{2}\right) \\
-\frac{3}{\sqrt{6}} \mathbb{E}^{\oplus} S_{1} \mathbb{E}^{\oplus} S_{2} & \frac{1}{\sqrt{2}} \mathbb{E}^{\oplus} S_{2}\left(\mathbb{E}^{\oplus} S_{1}+2 \mathbb{E}^{\oplus} S_{3}\right) \\
-\frac{3}{\sqrt{6}} \mathbb{E}^{\oplus} S_{1} \mathbb{E}^{\oplus} S_{3} & -\frac{1}{\sqrt{2}} \mathbb{E}^{\oplus} S_{3}\left(\mathbb{E}^{\oplus} S_{1}+2 \mathbb{E}^{\oplus} S_{2}\right)
\end{array}\right] \\
= & {\left[\mathbf{S}_{\mathbf{i t}}\right] \odot\left[\begin{array}{cc}
\frac{3}{\sqrt{6}}\left(1-\mathbb{E}^{\oplus} S_{1}\right) & \frac{1}{\sqrt{2}}\left(\mathbb{E}^{\oplus} S_{3}-\mathbb{E}^{\oplus} S_{2}\right) \\
-\frac{3}{\sqrt{6}} \mathbb{E}^{\oplus} S_{1} & \frac{1}{\sqrt{2}}\left(\mathbb{E}^{\oplus} S_{1}+2 \mathbb{E}^{\oplus} S_{3}\right) \\
-\frac{3}{\sqrt{6}} \mathbb{E}^{\oplus} S_{1} & -\frac{1}{\sqrt{2}}\left(\mathbb{E}^{\oplus} S_{1}+2 \mathbb{E}^{\oplus} S_{2}\right)
\end{array}\right]=\left[\mathbf{S}_{\mathbf{i t}}\right] \odot \mathbf{W}^{*} }
\end{aligned}
$$


The Jacobian of the model in the simplex $J$

$$
\begin{aligned}
J & =J_{T^{-1}} J_{F} J_{T}=\left[\begin{array}{lll}
\frac{\partial S_{1}}{\partial \dot{X}_{1}} & \frac{\partial S_{1}}{\partial \dot{X}_{2}} & \frac{\partial S_{1}}{\partial \dot{X}_{3}} \\
\frac{\partial S_{2}}{\partial \dot{X}_{1}} & \frac{\partial S_{2}}{\partial \dot{X}_{2}} & \frac{\partial S_{2}}{\partial \dot{X}_{3}} \\
\frac{\partial S_{3}}{\partial \check{X}_{1}} & \frac{\partial S_{3}}{\partial \check{X}_{2}} & \frac{\partial S_{3}}{\partial \check{X}_{3}}
\end{array}\right] \\
& =\left[\mathbf{S}_{\mathbf{i t}}\right] \odot \mathbf{W}^{*} \mathbf{B}^{*} \mathbf{V}^{\prime} \odot\left[\mathbf{1} / \check{\mathbf{X}}_{\mathbf{j}}\right]=\left[\mathbf{S}_{\mathbf{i t}}\right] \odot \mathbf{W}^{*} \mathbf{V}^{\prime} \mathbf{B} \odot\left[\mathbf{1} / \check{\mathbf{X}}_{\mathbf{j}}\right]=\left[\mathbf{S}_{\mathbf{i t}}\right] \odot \mathbf{W B} \odot\left[\mathbf{1} / \check{\mathbf{X}}_{\mathbf{j}}\right] \\
& =\left[\mathbf{S}_{\mathbf{i t}}\right] \odot\left[\begin{array}{cc}
\frac{3}{\sqrt{6}}\left(1-\mathbb{E}^{\oplus} S_{1}\right) & \frac{1}{\sqrt{2}}\left(\mathbb{E}^{\oplus} S_{3}-\mathbb{E}^{\oplus} S_{2}\right) \\
-\frac{3}{\sqrt{6}} \mathbb{E}^{\oplus} S_{1} & \frac{1}{\sqrt{2}}\left(\mathbb{E}^{\oplus} S_{1}+2 \mathbb{E}^{\oplus} S_{3}\right) \\
-\frac{3}{\sqrt{6}} \mathbb{E}^{\oplus} S_{1} & -\frac{1}{\sqrt{2}}\left(\mathbb{E}^{\oplus} S_{1}+2 \mathbb{E}^{\oplus} S_{2}\right)
\end{array}\right]\left[\begin{array}{ll}
b_{11}^{*} & b_{12}^{*} \\
b_{21}^{*} & b_{22}^{*}
\end{array}\right]\left[\begin{array}{ccc}
\sqrt{\frac{2}{3}} & -\frac{1}{\sqrt{6}} & -\frac{1}{\sqrt{6}} \\
0 & \frac{1}{\sqrt{2}} & -\frac{1}{\sqrt{2}}
\end{array}\right] \odot\left[\mathbf{1} / \check{\mathbf{X}}_{\mathbf{j}}\right] \\
& =\left[\mathbf{S}_{\mathbf{i t}}\right] \odot\left[\begin{array}{ccc}
1-S_{1} & -S_{2} & -S_{3} \\
-S_{1} & 1-S_{2} & -S_{3} \\
-S_{1} & -S_{2} & 1-S_{3}
\end{array}\right]\left[\begin{array}{lll}
b_{11} & b_{12} & b_{13} \\
b_{21} & b_{22} & b_{23} \\
b_{31} & b_{32} & b_{33}
\end{array}\right] \odot\left[\mathbf{1} / \check{\mathbf{X}}_{\mathbf{j}}\right]=M E\left(\mathbb{E}^{\oplus} \mathbf{S}_{t}, \check{\mathbf{X}}_{t}\right) \\
& \Leftrightarrow E\left(\mathbb{E}^{\oplus} \mathbf{S}_{t}, \check{\mathbf{X}}_{t}\right)=\left[\begin{array}{cc}
\mathbf{1} \\
\mathbf{S}_{\mathbf{i t}}
\end{array}\right] \odot M E\left(\mathbb{E}^{\oplus} \mathbf{S}_{t}, \check{\mathbf{X}}_{t}\right) \odot\left[\check{\mathbf{X}}_{\mathbf{j}}\right]=\mathbf{W B}
\end{aligned}
$$

where $\mathbf{W}^{*} \mathbf{V}^{\prime}=\mathbf{W}$ is a $D, D$ matrix with $1-S_{i}$ in the diagonal and $-S_{i}$ in the row $i$ otherwise.

We then conclude that marginal effects and elasticities matrices are easy to compute using coefficients in the simplex or coefficients in the transformed space:

$$
\begin{aligned}
M E\left(\mathbb{E}^{\oplus} \mathbf{S}_{t}, \check{\mathbf{X}}_{t}\right) & =\left[\mathbf{S}_{\mathbf{i t}}\right] \odot \mathbf{W B} \odot\left[\mathbf{1} / \check{\mathbf{X}}_{\mathbf{j}}\right]=\left[\mathbf{S}_{\mathbf{i t}}\right] \odot \mathbf{W} \mathbf{V B} \mathbf{B}^{*} \mathbf{V}^{\prime} \odot\left[\mathbf{1} / \check{\mathbf{X}}_{\mathbf{j}}\right] \\
E\left(\mathbb{E}^{\oplus} \mathbf{S}_{t}, \check{\mathbf{X}}_{t}\right) & =\mathbf{W B}=\mathbf{W} \mathbf{V} \mathbf{B}^{*} \mathbf{V}^{\prime}
\end{aligned}
$$

\section{A.3. C derivatives}

We keep here the notations of chapter 13 in Pawlowsky-Glahn and Buccianti (2011) except that we denote $\frac{\partial f}{\partial \oplus_{x}}$ the part-C derivatives. Let $f$ be a vector valued scale invariant function from $\mathbb{R}^{D_{X}}$ to $\mathbb{R}^{k}$. Let $f$ be the corresponding vector valued function on $\mathcal{S}^{D_{X}}$ induced by $f(\mathbf{x})=f(\mathbf{w})$, where $\mathbf{w}$ is the vector of volumes corresponding to the vector of shares $\mathbf{x}$. We have

$$
f(w)=\underline{f}(\mathcal{C}(\mathbf{w}))
$$

For the sake of simplicity, let us assume that $D_{X}=3$. Denote by $w_{+}=\sum_{i=1}^{D_{X}} w_{i}$ the total volume. Taking the derivative of (21) with respect to $w_{j}$ yields

$$
\frac{\partial f}{\partial w_{j}}(\mathbf{w})=\sum_{i=1}^{3} \frac{\partial f}{\partial x_{i}}(\mathbf{x}) \frac{\partial x_{i}}{\partial w_{j}}
$$

Since $\frac{\partial x_{i}}{\partial w_{i}}=\frac{w_{+}-w_{i}}{w_{+}^{2}}$ and $\frac{\partial x_{i}}{\partial w_{j}}=\frac{-w_{i}}{w_{+}^{2}}$ if $i \neq j$, we obtain

$$
\begin{aligned}
\frac{\partial f}{\partial w_{j}}(\mathbf{w}) & =\frac{1}{w_{+}^{2}}\left[w_{+} \frac{\partial \underline{f}}{\partial x_{j}}(\mathbf{x})-\sum_{i=1}^{3} w_{i} \frac{\partial \underline{f}}{\partial x_{i}}(\mathbf{x})\right] \\
& =\frac{1}{w_{+}}\left[\frac{\partial \underline{f}}{\partial x_{j}}(\mathbf{x})-\sum_{i=1}^{3} x_{i} \frac{\partial \underline{f}}{\partial x_{i}}(\mathbf{x})\right]
\end{aligned}
$$

Proposition 13.3.5 tells us that

$$
\frac{\partial \underline{f}}{\partial^{\oplus} x_{j}}(\mathbf{x})=x_{j}\left[\frac{\partial \underline{f}}{\partial x_{j}}(\mathbf{x})-\sum_{i=1}^{3} x_{i} \frac{\partial \underline{f}}{\partial x_{i}}(\mathbf{x})\right]
$$


Using (22) with $w_{j}$ replaced by $\log \left(w_{j}\right)$ yields

$$
\frac{\partial f}{\partial \log \left(w_{j}\right)}(\mathbf{w})=w_{j} \frac{\partial f}{\partial w_{j}}(\mathbf{w})=\frac{w_{j}}{w_{+}}\left[\frac{\partial \underline{f}}{\partial x_{j}}(\mathbf{x})-\sum_{i=1}^{3} x_{i} \frac{\partial \underline{f}}{\partial x_{i}}(\mathbf{x})\right]=x_{j}\left[\frac{\partial \underline{f}}{\partial x_{j}}(\mathbf{x})-\sum_{i=1}^{3} x_{i} \frac{\partial \underline{f}}{\partial x_{i}}(\mathbf{x})\right]
$$

Combining this with (24) yields the following proposition linking the semi-log derivatives of $f$ with the directional $\mathcal{C}$ derivatives of $\underline{f}$.

$$
\frac{\partial \underline{f}}{\partial^{\oplus} x_{j}}(\mathbf{x})=\frac{\partial f}{\partial \log \left(w_{j}\right)}(\mathbf{w})
$$

Let us now consider the case of a function from the simplex $\mathcal{S}^{D_{X}}$ of $\mathbb{R}^{D_{X}}$ to the simplex $\mathcal{S}^{D_{S}}$ of $\mathbb{R}^{D_{S}}$. Rewriting (12.6) from chapter 12 (page 163) with our present notations we have

$$
\frac{\partial^{\oplus} \mathbf{h}}{\partial t}(t)=\mathcal{C} \exp \left(\frac{\partial \log (\mathbf{h})}{\partial t}\right) .
$$

Combining this with (25), we can define the following directional $\mathcal{C}$ derivatives of $\mathbf{h}$, denoted $\frac{\partial^{\oplus} \mathbf{h}}{\partial^{\oplus} x_{j}}$ as $\frac{\partial^{\oplus} \mathbf{h}}{\partial^{\oplus} x_{j}}=\mathcal{C} \exp \left(\frac{\partial \log (\mathbf{h})}{\partial^{\oplus} x_{j}}\right)=\mathcal{C} \exp \left(\frac{\partial \log (\mathbf{h})}{\partial \log w_{j}}\right)$.

\section{A.4. Nullity of the sum of elasticities weighted by shares}

We have to prove that $\sum_{m=1}^{D} e_{m l t} \mathbb{E}^{\oplus} S_{m t}=0$. This is the necessary condition for new shares $S_{m t}^{\prime}$, resulting from a change in $X_{l t}$, to sum up to one: $\sum_{m=1}^{D} S_{m t}^{\prime}=1 \Leftrightarrow \sum_{m=1}^{D} e_{m l t} \mathbb{E}^{\oplus} S_{m t}=$ 0 .

Proof:

$\sum_{m=1}^{D} \mathbb{E}^{\oplus} S_{m t}=1 \Leftrightarrow \sum_{m=1}^{D} \frac{\partial \mathbb{E}^{\oplus} S_{m t}}{\partial \log X_{l t}}=0 \Leftrightarrow \sum_{m=1}^{D} \frac{\partial \mathbb{E}^{\oplus} S_{m t}}{\partial \log X_{l t}} \frac{1}{\mathbb{E}^{\oplus} S_{m t}} \mathbb{E}^{\oplus} S_{m t}=0 \Leftrightarrow \sum_{m=1}^{D} e_{m l t} \mathbb{E}^{\oplus} S_{m t}=0$

\section{Affiliation:}

Joanna Morais

Toulouse School of Economics

21 allée de Brienne

31000 Toulouse, France

E-mail: joanna.morais@live.fr

URL: https://www.linkedin.com/in/joanna-morais-77704867

\section{Austrian Journal of Statistics}

published by the Austrian Society of Statistics

Volume 47

September 2018 http://www .ajs.or.at/

http://www.osg.or.at/

Submitted: 2017-07-19

Accepted: 2018-04-23 